\title{
A Fuzzy Trustworthiness System with Probability Presentation Based on Center-of-gravity Method
}

\author{
Yu-Bin Zhong ${ }^{1,2}$ (D) Zeng-Liang Liu ${ }^{3}$ • \\ Xue-Hai Yuan 4
}

Received: 7 December 2015 / Revised: 8 December 2015 / Accepted: 9 December 2015 /

Published online: 28 January 2016

(C) Springer-Verlag Berlin Heidelberg 2016

\begin{abstract}
Fuzzy methods are widely used in the study of trustworthiness. Based on this fact, the paper researches the fuzzy trustworthiness system and probability presentation theory based on bounded product implication and Larsen square implication. Firstly, we convert a group of single-input and single-output data into fuzzy inference rules and generate fuzzy relation by selecting the appropriate fuzzy implication operator, then calculate joint probability density function of two-dimensional random variables by using of this fuzzy relation. Two specific probability density functions can be obtained by selecting the fuzzy implication as bounded product implication or Larsen square implication. Secondly, we study the marginal distribution and numerical characteristics of these two kinds probability distributions and point out that two of these probability distributions have the same mathematical expectation and nearly the same variance and covariance. Finally, we study of the center-of-gravity fuzzy trustworthiness system
\end{abstract}

$凶 \quad$ Yu-Bin Zhong

zhong_yb@163.com

Zeng-Liang Liu

chinapd@tom.com

Xue-Hai Yuan

yuanxuehai@yahoo.com.cn

1 College of Mathematics and Information Sciences, Guangzhou University Guangzhou, Guangdong 510006, People's Republic of China

2 Key Laboratory of Mathematics and Interdisciplinary Sciences of Guangdong Higher Education Institutes, Guangzhou University, Guangzhou, China

3 Institute of Information Operation National Defence, University of PLA, Beijing 100091, People's Republic of China

4 Faculty of Electronic Information and Electrical Engineering, Dalian University of Technology, Dalian 116024, China 
based on these two probability distributions. We gave the sufficient conditions of universal approximations for those fuzzy trustworthiness systems.

Keywords Fuzzy trustworthiness system - Probability density function - Numerical characteristics · Universal approximation · Application research

\section{Introduction}

Fuzziness, widely existing in real life and various systems, is a kind of uncertainty that is entirely different from contingency [1]. Fuzzy methods have wide application backgrounds in the study of trustworthiness, for example, (1) in fuzzy systems, the input, output and their cross linking state could be fuzzy; (2) in human behaviors, there also exists various kinds of fuzziness; (3) a software is unique, and trustworthiness behaviors of software are fuzzy in nature; (4) in control systems, we may have fuzzy control, design trustworthiness and intelligent control, etc. ; (5) in expert system research, it is necessary to take into consideration of the consistency, completeness, independence and redundancy of knowledge bases, but it is also important to consider fuzzy expert systems and the spread of non-trustworthiness factors. Therefore, it is significant to study the fuzzy trustworthiness system and its probability presentation theory, and this will benefit a lot to production and life practices.

Construction of fuzzy trustworthiness systems and the universal approximation of them are hot topics in the research of fuzzy system and fuzzy control theory. The most familiar construction of fuzzy trustworthiness system is divided into four processes [2]: (1) fuzzifier of the input variables; (2) construct the inference relationship; (3) fuzzy inference; (4) defuzzifier of the output variables set.

In the construction of fuzzy trustworthiness systems, the single fuzzifier of the input variable has been mentioned in most literatures until now. And fuzzy inference is CRI reasoning method $[3,4]$ or triple I method [5]. We must determine the fuzzy implication operator before constructing fuzzy inference relationship and fuzzy reasoning. The choice of fuzzy implication operator will have a huge impact on the fuzzy trustworthiness system. References [6-9] point out that, CRI reasoning method and triple I method have impact on the fuzzy trustworthiness system when only using the conjunction type fuzzy implication operators, such as Mamdani implication and Larsen implication. The common defuzzification methods are: (a) center-average defuzzification method, (b) center-of-gravity defuzzification method, and (c) maximum defuzzification method. Researchers have constructed fuzzy systems by using the center-average defuzzification method [10-14] and maximum defuzzification method [15-17] until now. Because of involved some complex integral, researchers have not yet given the specific expression of the fuzzy trustworthiness system when constructing them by using center-of-gravity method. How to obtain the specific expression of this fuzzy trustworthiness system is one motivation in our paper.

As we know, the fuzzy trustworthiness system constructed by center-of-gravity method can be approximately reduced to some forms of interpolation [18] and this fuzzy trustworthiness system has meaning of probability theory. It is the best approximation of system under the sense of least-squares [19]. But how to determine the 
corresponding probability density function of fuzzy implication operator has not been resolved, and this is the another motivation in our paper.

In order to obtain the corresponding probability density function of fuzzy implication operator, we are starting from the input and output data and through restrictions on the fuzzy reasoning relationship then we succeeded in getting the corresponding probability density function of bounded product implication and Larsen square implication. We found that adopting different fuzzy implication operators will obtain different probability density functions, but the corresponding random variables have the same mathematical expectation and almost the same variance and covariance. Further, we derived the corresponding center-of-gravity fuzzy trustworthiness systems by using these two kinds of probability distributions and gave the sufficient conditions of universal approximation for them.

This article is organized as follows: Sect. 2 is Preliminary; Sect. 3 is the probability distributions and marginal probability distribution of bounded product implication and Larsen square implication; Sect. 4 discusses the numerical characteristic of these two distributions; Sect. 5 gives the center-of-gravity method fuzzy trustworthiness systems of these two probability distributions and the sufficient condition with universal approximation for these systems ; Sect. 6 is a overlook of application analysis of fuzzy trustworthiness systems and we have our conclusion in Sect. 7.

\section{Preliminary}

Suppose $\left\{\left(x_{i}, y_{i}\right)\right\}_{(1 \leq i \leq n)}$ is a group of input-output data, then

$$
a=x_{1}<x_{2}<\cdots<x_{n}=b, \quad c=y_{1}<y_{2}<\cdots<y_{n}=d,
$$

or

$$
a=x_{1}<x_{2}<\cdots<x_{n}=b, \quad c=y_{n}<y_{n-1}<\cdots<y_{1}=d .
$$

we construct a two-phase triangular wave by using these data, namely

$$
A_{1}(x)=\left\{\begin{array}{l}
\frac{x_{2}-x}{x_{2}-x_{1}}, \quad x \in\left[x_{1}, x_{2}\right] \\
0, \text { other }
\end{array} \quad, \quad A_{n}(x)=\left\{\begin{array}{l}
\frac{x_{n}-x}{x_{n}-x_{n-1}}, \quad x \in\left[x_{1}, x_{2}\right], \\
0, \text { other }
\end{array}\right.\right.
$$

for $i=2,3, \ldots, n-1$,

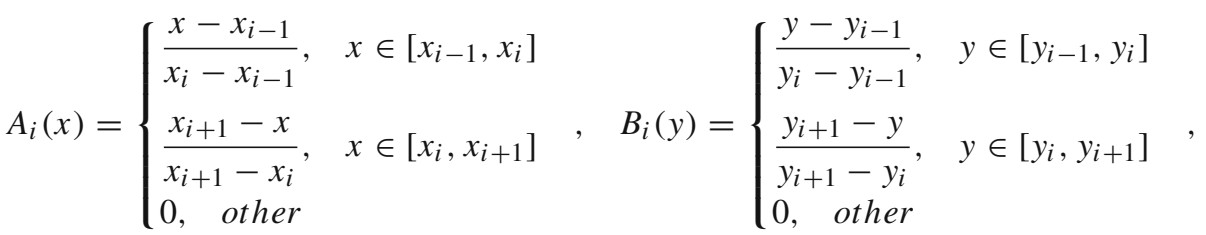

$$
\begin{aligned}
& B_{1}(y)=\left\{\begin{array}{l}
\frac{y_{2}-y}{y_{2}-y_{1}}, \quad y \in\left[y_{1}, y_{2}\right] \\
0, \text { other }
\end{array} \quad, \quad B_{n}(y)=\left\{\begin{array}{l}
\frac{y_{n}-y}{y_{n}-y_{n-1}}, \quad y \in\left[y_{n-1}, y_{n}\right] \\
0, \text { other }
\end{array},\right.\right.
\end{aligned}
$$


then $x_{i}, y_{i}$ is the peak point of $A_{i}$ and $y_{i}$, namely $A_{i}\left(x_{i}\right)=1, B_{i}\left(y_{i}\right)=1,(i=$ $1,2, \ldots, n)$, and

when $x \in\left[x_{i}, x_{i+1}\right], \quad A_{i}(x)+A_{i+1}(x)=1, \quad A_{j}(x)=0(j \neq i, i+1)$,

when $y \in\left[y_{i}, y_{i+1}\right], \quad B_{i}(y)+B_{i+1}(y)=1, \quad B_{j}(y)=0(j \neq i, i+1)$.

Then we have fuzzy inference rule

$$
\text { if } x \text { is } A_{i}, \quad \text { then } y \text { is } B_{i} \quad(i=1,2, \ldots, n) .
$$

Let $\theta$ be the fuzzy implication operator, then from (1) can get the fuzz relationship $R(x, y)=\vee_{i=1}^{n} \theta\left(A_{i}(x), B_{i}(y)\right)$.

Let $q(x, y)=\left\{\begin{array}{l}R(x, y),(x, y) \in X \times Y \\ 0, \quad \text { other }\end{array} \quad\right.$, and $H(2, n, \theta, \vee) \triangleq \int_{-\infty}^{+\infty} \int_{-\infty}^{+\infty} q(x, y)$ $d x d y$, when $X=[a, b], Y=[c, d]$, we have

$$
H(2, n, \theta, \vee)=\int_{a}^{b} \int_{c}^{d} R(x, y) d x d y
$$

we call $H(2, n, \theta, \vee) H$ function which has parameter $2, n, \theta, \vee$, and " 2 " indicates $q(x, y)$ is binary function; $n$ means the amount of inference rules; $\theta$ is fuzzy implication operator, and $\vee=$ "max".

If $H(2, n, \theta, \vee)>0$, let

$$
f(x, y) \triangleq \frac{q(x, y)}{H(2, n, \theta, \vee)}
$$

clearly, (a) $f(x, y) \geq 0$; (b) $\int_{-\infty}^{+\infty} \int_{-\infty}^{+\infty} f(x, y) d x d y=1$. So, $f(x, y)$ can be regarded as a random vector of the joint probability density function $(\xi, \eta)$ [19].

Suppose $R(x, y)$ is fuzzy relationship determined by inference rules (1), and $A^{*}(x)$ is a fuzzy single point of input variables, namely $A^{*}\left(x^{\prime}\right)=\left\{\begin{array}{l}1, x^{\prime}=x \\ 0, x^{\prime} \neq x\end{array}\right.$. Let $B^{*}=$ $A \circ R$, namely $B^{*}(y)=\underset{x^{\prime} \in X}{\vee}\left(A^{*}\left(x^{\prime}\right) \wedge R\left(x^{\prime}, y\right)\right)=R(x, y)$. Then

$$
\bar{S}(x)=\frac{\int_{c}^{d} y B^{*}(y) d y}{\int_{c}^{d} B^{*}(y) d y}
$$

is a center-of-gravity method fuzzy trustworthiness system [18].

\section{The Probability Distribution of Several Single-Input and Single-Output Fuzzy Trustworthiness System}

If $\left\{\left(x_{i}, y_{i}\right)\right\}_{1 \leq i \leq n}$ satisfies:

$$
a=x_{1}<x_{2}<\cdots<x_{n}=b, \quad c=y_{1}<y_{2}<\cdots<y_{n}=d .
$$


Establishing fuzzy relations according to the fuzzy inference rule (1) is one of the four processes of constructing a fuzzy trustworthiness system. If using the traditional method, then the fuzzy relation is $R(x, y)=\vee_{i=1}^{n} \theta\left(A_{i}(x), B_{i}(y)\right)$. Since the fuzzy implication is conjunction implication, we have the following result:

if $x \in\left[x_{i}, x_{i+1}\right]$, then $R(x, y)=\theta\left(A_{i}(x), B_{i}(y)\right) \vee \theta\left(A_{i+1}(x), B_{i+1}(y)\right)$.

Because the data is monotonic, we make some adjustments to the fuzzy reasoning relationship: when $x \in\left[x_{i}, x_{i+1}\right]$, let

$$
R(x, y)=\left\{\begin{array}{l}
\theta\left(A_{i}(x), B_{i}(y)\right) \vee \theta\left(A_{i+1}(x), B_{i+1}(y)\right), \quad y \in\left[y_{i}, y_{i+1}\right] \\
0, \quad \text { other. }
\end{array}\right.
$$

We discuss the reasoning relationship determine by (5), then we have the following probability results:

Theorem 1 If $\theta(a, b)=T_{m}(a, b)=(a+b-1) \vee 0$, let $H=\frac{1}{3} \sum_{i=1}^{n-1}\left(x_{i+1}-\right.$ $\left.x_{i}\right)\left(y_{i+1}-y_{i}\right) \cdot D_{i}=\left\{(x, y) \in I_{i} \times J_{i} \mid y \leq A_{i+1}(x) y_{i}+A_{i}(x) y_{i+1}\right\}, E_{i}=I_{i} \times J_{i}-D_{i}$, then

(1) Probability density function $f(x, y)$ (called Bounded plot distribution) determined by formula (3) is

$$
f(x, y)=\left\{\begin{array}{l}
\frac{1}{H}\left(A_{i}(x)+B_{i}(y)-1\right), \quad(x, y) \in D_{i} \\
\frac{1}{H}\left(A_{i+1}(x)+B_{i+1}(y)-1\right), \quad(x, y) \in E_{i} \\
0, \quad \text { other }
\end{array}\right.
$$

(2) Marginal distribution functions of $f(x, y)$ are

$$
\begin{aligned}
& f_{\xi}(x)=\frac{1}{2 T}\left(y_{i+1}-y_{i}\right)\left(1-2 A_{i}(x) A_{i+1}(x)\right), \quad x \in\left[x_{i}, x_{i+1}\right](i=1,2, \ldots n) . \\
& f_{\eta}(x)=\frac{1}{2 T}\left(x_{i+1}-x_{i}\right)\left(1-2 B_{i}(y) B_{i+1}(y)\right), \quad y \in\left[y_{i}, y_{i+1}\right](i=1,2, \ldots n) .
\end{aligned}
$$

Proof (1) Because

$$
\begin{aligned}
B^{*}(y) & =\left(A_{i}(x)+B_{i}(y)-1\right) \vee\left(A_{i+1}(x)+B_{i+1}(y)-1\right) \vee 0 \\
& =\left(A_{i}(x)+B_{i}(y)-1\right) \vee\left(A_{i+1}(x)+B_{i+1}(y)-1\right),
\end{aligned}
$$

then

$$
\begin{aligned}
H(2, n, \theta, \vee)= & \sum_{i=1}^{n-1} \int_{x_{i}}^{x_{i+1}} \int_{y_{i}}^{y_{i+1}}\left[( A _ { i } ( x ) + B _ { i } ( y ) - 1 ) \vee \left(A_{i+1}(x)\right.\right. \\
& \left.\left.+B_{i+1}(y)-1\right)\right] d y d x .
\end{aligned}
$$


If $A_{i}(x)+B_{i}(y) \geq 1$, then $A_{i+1}(x)+B_{i+1}(y) \leq 1$ and $B_{i}(y) \geq 1-A_{i}(x)=A_{i+1}(x)$, namely $\frac{y_{i+1}-y}{y_{i+1}-y_{i}} \geq A_{i+1}(x)$. Then $y \leq A_{i+1}(x) y_{i}+A_{i}(x) y_{i+1} \triangleq y_{i}^{*}$. So

$$
\begin{aligned}
\triangle_{i} & =\int_{x_{i}}^{x_{i+1}} \int_{y_{i}}^{y_{i+1}}\left[\left(A_{i}(x)+B_{i}(y)-1\right) \vee\left(A_{i+1}(x)+B_{i+1}(y)-1\right)\right] d y d x \\
& =\int_{x_{i}}^{x_{i+1}}\left[\int_{y_{i}}^{y_{i}^{*}}\left(A_{i}(x)+B_{i}(y)-1\right) d y+\int_{y_{i}^{*}}^{y_{i+1}}\left(A_{i+1}(x)+B_{i+1}(y)-1\right) d y\right] d x \\
& =\left(y_{i+1}-y_{i}\right) \int_{x_{i}}^{x_{i+1}}\left(\frac{1}{2}-A_{i}(x) A_{i+1}(x)\right) d x .
\end{aligned}
$$

Let $A_{i+1}(x)=\frac{x-x_{i}}{x_{i+1}-x_{i}}=t$, then $A_{i}(x)=1-t, d x=\left(x_{i+1}-x_{i}\right) d t$. So

$$
\Delta_{i}=\left(x_{i+1}-x_{i}\right)\left(y_{i+1}-y_{i}\right) \int_{0}^{1}\left[\frac{1}{2}-t(1-t)\right] d t=\frac{1}{3}\left(x_{i+1}-x_{i}\right)\left(y_{i+1}-y_{i}\right)
$$

Then $H(2, n, \theta, \vee)=\frac{1}{3} \sum_{i=1}^{n-1}\left(x_{i+1}-x_{i}\right)\left(y_{i+1}-y_{i}\right) \triangleq H$. And we have,

$$
f(x, y)=\left\{\begin{array}{l}
\frac{1}{H}\left(A_{i}(x)+B_{i}(y)-1\right), \quad \exists i,(x, y) \in D_{i} \\
\frac{1}{H}\left(A_{i+1}(x)+B_{i+1}(y)-1\right), \quad \exists i,(x, y) \in E_{i} \\
0, \quad \text { other }
\end{array}\right.
$$

(2)

$$
\begin{aligned}
f_{\xi}(x)= & \frac{1}{T}\left[\int_{y_{i}}^{y_{i}^{*}}\left(A_{i}(x)+B_{i}(y)-1\right) d y+\int_{y_{i}^{*}}^{y_{i+1}}\left(A_{i+1}(x)+B_{i+1}(y)-1\right) d y\right] \\
= & \frac{1}{T}\left[\left(A_{i}(x)-1\right)\left(y_{i}^{*}-y_{i}\right)+\int_{y_{i}}^{y_{i}^{*}} B_{i}(y) d y+\left(A_{i+1}(x)-1\right)\left(y_{i+1}-y_{i}^{*}\right)\right. \\
& \left.+\int_{y_{i}^{*}}^{y_{i+1}} B_{i+1}(y) d y\right] .
\end{aligned}
$$

Because $y_{i}^{*}-y_{i}=\left(y_{i+1}-y_{i}\right) A_{i}(x), y_{i+1}-y_{i}^{*}=\left(y_{i+1}-y_{i}\right) A_{i+1}(x)$, we make convert $B_{i+1}(y)=\frac{y-y_{i}}{y_{i+1}-y_{i}}=t$, then we get 


$$
\begin{aligned}
f_{\xi}(x)= & \frac{y_{i+1}-y_{i}}{T}\left[\left(A_{i}(x)-1\right) A_{i}(x)+\int_{0}^{A_{i}(x)}(1-t) d t+\left(A_{i+1}(x)-1\right) A_{i+1}(x)\right. \\
& \left.+\int_{A_{i}(x)}^{1} t d t\right] \\
= & \frac{y_{i+1}-y_{i}}{T}\left[A_{i}^{2}-A_{i}(x)+A_{i}(x)-\frac{1}{2} A_{i}^{2}(x)+A_{i+1}^{2}(x)-A_{i+1}^{2}(x)\right. \\
& \left.+\frac{1}{2}\left(1-A_{i}^{2}(x)\right)\right] \\
= & \frac{y_{i+1}-y_{i}}{2 T}\left[1-2 A_{i}(x) A_{i+1}(x)\right] .
\end{aligned}
$$

The others can be proved similarly.

Theorem 2 If $\theta(a, b)=a^{2} b$, then

(1) Probability density function $f(x, y)$ (called Larsen square distribution) determined by formula (3) is

$$
f(x, y)=\left\{\begin{array}{l}
\frac{1}{C} A_{i}^{2}(x) B_{i}(y), \quad \exists i,(x, y) \in F_{i} \\
\frac{1}{C} A_{i+1}^{2}(x) B_{i+1}(y), \quad \exists i,(x, y) \in G_{i} \\
0, \quad \text { other }
\end{array}\right.
$$

(2) Marginal density function of $f(x, y)$ is: for $x \in\left[x_{i}, x_{i+1}\right)(i=1,2, \ldots, n)$

$$
\begin{aligned}
& f_{\xi}(x)=\frac{1}{2 C}\left(y_{i+1}-y_{i}\right) \frac{\left(1-A_{i}(x) A_{i+1}(x)\right)\left(1-3 A_{i}(x) A_{i+1}(x)\right)}{1-A_{i}(x) A_{i+1}(x)}, \\
& f_{\eta}(y)=\frac{1}{3 C}\left(x_{i+1}-x_{i}\right)\left(1-\frac{B_{i}(y) B_{i+1}(y)}{1+2 \sqrt{B_{i}(y) B_{i+1}(y)}}\right)\left(y \in\left[y_{i}, y_{i+1}\right]\right),
\end{aligned}
$$

$$
\begin{aligned}
& \text { where } F_{i}=\left\{(x, y) \in I_{i} \times J_{i} \mid y \leq \frac{A_{i+1}^{2}(x)}{A_{i}^{2}(x)+A_{i+1}^{2}(x)} y_{i}+\frac{A_{i}^{2}(x)}{A_{i}^{2}(x)+A_{i+1}^{2}(x)} y_{i+1}\right\}, \\
& G_{i}=\left(I_{i} \times J_{i}\right)-F_{i}, K=\frac{1}{2}-\frac{1}{16} \pi, \text { and } C=K \sum_{i=1}^{n-1}\left(x_{i+1}-x_{i}\right)\left(y_{i+1}-y_{i}\right) .
\end{aligned}
$$

Proof (1) $H(2, n, \theta, \vee)=\int_{a}^{b} \int_{c}^{d} p(x, y) d y d x$, here when $x \in\left[x_{i}, x_{i+1}\right]$, we have $p(x, y)=A_{i}^{2}(x) B_{i}(y) \vee A_{i+1}^{2}(x) B_{i+1}(y)$.

Then $H(2, n, \theta, \vee)=\sum_{i=1}^{n-1} \int_{x_{i}}^{x_{i+1}} \int_{y_{i}}^{y_{i+1}}\left(A_{i}^{2}(x) B_{i}(y) \vee A_{i+1}^{2}(x) B_{i+1}(y)\right) d y d x$. then

For $x \in\left[x_{i}, x_{i+1}\right]$, when $A_{i}^{2}(x) B_{i}(y) \geq A_{i+1}^{2}(x) B_{i+1}(y)=A_{i}^{2}(x)\left(1-B_{i}(y)\right)$,

$$
B_{i}(y)=\frac{y_{i+1}-y}{y_{i+1}-y_{i}} \geq \frac{A_{i+1}^{2}(x)}{A_{i}^{2}(x)+A_{i+1}^{2}(x)}
$$


so

$$
y \leq \frac{A_{i+1}^{2}(x)}{A_{i}^{2}(x)+A_{i+1}^{2}(x)} y_{i}+\frac{A_{i}^{2}(x)}{A_{i}^{2}(x)+A_{i+1}^{2}(x)} y_{i+1} \triangleq \tilde{y}_{i} .
$$

Then $\Delta^{*}=\int_{y_{i}}^{y_{i+1}}\left(A_{i}^{2}(x) B_{i}(y) \vee A_{i+1}^{2} B_{i+1}(y)\right) d y=\int_{y_{i}}^{\tilde{y}_{i}} A_{i}^{2}(x) B_{i}(y) d y+\int_{\tilde{y}_{i}}^{y_{i+1}}$ $A_{i+1}^{2}(x) B_{i+1}(y) d y=A_{i}^{2}(x) \int_{y_{i}}^{\widetilde{y}_{i}} B_{i}(y) d y+A_{i+1}^{2}(x) \int_{\tilde{y}_{i}}^{y_{i+1}} B_{i+1}(y) d y$.

$$
\int_{y_{i}}^{\widetilde{y}_{i}} B_{i}(y) d y=\frac{1}{y_{i+1}-y_{i}}\left[y_{i+1} y-\frac{1}{2} y^{2}\right] \mid \tilde{y}_{y_{i}}=\frac{\left(\tilde{y}_{i}-y_{i}\right)\left(2 y_{i+1}-y_{i}-\tilde{y}_{i}\right)}{2\left(y_{i+1}-y_{i}\right)} .
$$

And

$$
\begin{aligned}
\tilde{y}_{i}-y_{i}= & \left(\frac{A_{i+1}^{2}(x)}{A_{i}^{2}(x)+A_{i+1}^{2}(x)}-1\right) y_{i}+\frac{A_{i}^{2}(x)}{A_{i}^{2}(x)+A_{i+1}^{2}(x)} y_{i+1} \\
= & \frac{A_{i}^{2}(x)}{A_{i}^{2}(x)+A_{i+1}^{2}(x)}\left(y_{i+1}-y_{i}\right) . \\
& 2 y_{i+1}-y_{i}-\tilde{y}_{i}=\left(y_{i+1}-y_{i}\right)+y_{i+1}-\tilde{y}_{i}=y_{i+1}-y_{i} \\
& +\frac{A_{i+1}^{2}(x)}{A_{i}^{2}(x)+A_{i+1}^{2}(x)}\left(y_{i+1}-y_{i}\right) \\
= & \frac{A_{i}^{2}(x)+2 A_{i+1}^{2}(x)}{A_{i}^{2}(x)+A_{i+1}^{2}(x)}\left(y_{i+1}-y_{i}\right) .
\end{aligned}
$$

So

$$
\begin{aligned}
\int_{y_{i}}^{\widetilde{y}_{i}} B_{i}(y) d y & =\frac{A_{i}^{2}(x)\left(A_{i}^{2}(x)+2 A_{i+1}^{2}(x)\right)}{2\left(A_{i}^{2}(x)+A_{i+1}^{2}(x)\right)^{2}}\left(y_{i+1}-y_{i}\right) . \\
\int_{\widetilde{y}_{i}}^{y_{i+1}} B_{i}(y) d y & =\frac{1}{y_{i+1}-y_{i}} \int_{\widetilde{y}_{i}}^{y_{i+1}}\left(y-y_{i}\right) d y=\frac{\left(y_{i+1}-\tilde{y}_{i}\right)\left(y_{i+1}-2 y_{i}+\tilde{y}_{i}\right)}{2\left(y_{i+1}-y_{i}\right)} .
\end{aligned}
$$

And

$$
\begin{aligned}
y_{i+1}-\tilde{y}_{i} & =\frac{A_{i+1}^{2}(x)}{A_{i}^{2}(x)+A_{i+1}^{2}(x)}\left(y_{i+1}-y_{i}\right) . \\
y_{i+1}-2 y_{i}+\tilde{y}_{i} & =\left(y_{i+1}-y_{i}\right)+\tilde{y}_{i}-y_{i}=\left(y_{i+1}-y_{i}\right)\left[1+\frac{A_{i}^{2}(x)}{A_{i}^{2}(x)+A_{i+1}^{2}(x)}\right] \\
& =\frac{2 A_{i}^{2}(x)+A_{i+1}^{2}(x)}{A_{i}^{2}(x)+A_{i+1}^{2}(x)}\left(y_{i+1}-y_{i}\right) .
\end{aligned}
$$


So $\int_{\tilde{y}_{i}}^{y_{i+1}} B_{i}(y) d y=\frac{A_{i+1}^{2}(x)\left(2 A_{i}^{2}(x)+A_{i+1}^{2}(x)\right)}{2\left(A_{i}^{2}(x)+A_{i+1}^{2}(x)\right)^{2}}\left(y_{i+1}-y_{i}\right)$.

Then

$$
\begin{aligned}
\Delta & =\left[\frac{A_{i}^{4}(x)\left(A_{i}^{2}(x)+2 A_{i+1}^{2}(x)\right)}{2\left(A_{i}^{2}(x)+A_{i+1}^{2}(x)\right)^{2}}+\frac{A_{i+1}^{4}(x)\left(2 A_{i}^{2}(x)+A_{i+1}^{2}(x)\right)}{2\left(A_{i}^{2}(x)+A_{i+1}^{2}(x)\right)^{2}}\right]\left(y_{i+1}-y_{i}\right) \\
& =\frac{y_{i+1}-y_{i}}{2} \bullet \frac{A_{i}^{6}(x)+A_{i+1}^{6}(x)+2 A_{i}^{2}(x) A_{i+1}^{2}(x)\left(A_{i}^{2}(x)+A_{i+1}^{2}(x)\right)}{\left(A_{i}^{2}(x)+A_{i+1}^{2}(x)\right)^{2}}
\end{aligned}
$$

so

$$
\begin{aligned}
\Delta^{*} & =\int_{x_{i}}^{x_{i+1}} \int_{y_{i}}^{y_{i+1}}\left(A_{i}^{2}(x) B_{i}(y) \vee A_{i+1}^{2}(x) B_{i+1}(y)\right) d y d x \\
& =\frac{y_{i+1}-y_{i}}{2} \int_{x_{i}}^{x_{i+1}} \frac{A_{i}^{6}(x)+A_{i+1}^{6}(x)+2 A_{i}^{2}(x) A_{i+1}^{2}(x)\left(A_{i}^{2}(x)+A_{i+1}^{2}(x)\right)}{\left(A_{i}^{2}(x)+A_{i+1}^{2}(x)\right)^{2}} d x .
\end{aligned}
$$

Let $A_{i+1}(x)=\frac{x-x_{i}}{x_{i+1}-x_{i}}=t$, then $d x=\left(x_{i+1}-x_{i}\right) d t, A_{i}(x)=1-t$. Then

$$
\Delta^{*}=\frac{\left(x_{i+1}-x_{i}\right)\left(y_{i+1}-y_{i}\right)}{2} \int_{0}^{1} \frac{t^{6}+(1-t)^{6}+2(1-t)^{2} t^{2}\left((1-t)^{2}+t^{2}\right)}{\left((1-t)^{2}+t^{2}\right)^{2}} d t
$$

Easy to calculate

$$
K=\frac{1}{2} \int_{0}^{1} \frac{t^{6}+(1-t)^{6}+2(1-t)^{2} t^{2}\left((1-t)^{2}+t^{2}\right)}{\left((1-t)^{2}+t^{2}\right)^{2}} d t=\frac{1}{2}-\frac{1}{16} \pi .
$$

Then $H(2, n, \theta, \vee)=K \sum_{i=1}^{n-1}\left(x_{i+1}-x_{i}\right)\left(y_{i+1}-y_{i}\right) \triangleq C$. So,

$$
f(x, y)=\left\{\begin{array}{l}
\frac{1}{C} A_{i}^{2}(x) B_{i}(y), \quad \exists i,(x, y) \in F_{i} \\
\frac{1}{C} A_{i+1}^{2}(x) B_{i+1}(y), \quad \exists i,(x, y) \in G_{i} \\
0, \quad \text { other }
\end{array}\right.
$$

here $F_{i}=\left\{(x, y) \in I_{i} \times J_{i} \mid y \leq \frac{A_{i+1}^{2}(x)}{A_{i}^{2}(x)+A_{i+1}^{2}(x)} y_{i}+\frac{A_{i}^{2}(x)}{A_{i}^{2}(x)+A_{i+1}^{2}(x)} y_{i+1}\right\}$, $G_{i}=\left(I_{i} \times J_{i}\right)-F_{i}$. 
(2)

$$
\begin{aligned}
f_{\xi}(x) & =\frac{1}{C}\left[\int_{y_{i}}^{\widetilde{y}_{i}} A_{i}^{2}(x) B_{i}(y) d y+\int_{\tilde{y}_{i}}^{y_{i+1}} A_{i+1}^{2}(x) B_{i+1}(y) d y\right] \\
& =\frac{1}{C}\left[A_{i}^{2}(x) \int_{y_{i}}^{\tilde{y}_{i}} B_{i}(y) d y+A_{i+1}^{2}(x) \int_{\tilde{y}_{i}}^{y_{i+1}} B_{i+1}(y) d y\right],
\end{aligned}
$$

where $\tilde{y}_{i}=\frac{A_{i+1}^{2}(x) y_{i}+A_{i}^{2}(x) y_{i+1}}{A_{i}^{2}(x)+A_{i+1}^{2}(x)}$. Let $B_{i+1}(y)=\frac{y-y_{i}}{y_{i+1}-y_{i}}=t$, then $d y=$ $\left(y_{i+1}-y_{i}\right) d t, B_{i}(y)=1-t$; when $y=y_{i}, t=0$; when $y=y_{i+1}, t=1$; when $y=\tilde{y}_{i}, t=\frac{A_{i}^{2}(x)}{A_{i}^{2}(x)+A_{i+1}^{2}(x)} \triangleq A_{i}^{*}(x)$.

$$
\begin{aligned}
& \text { Let } A_{i+1}^{*}(x)=\frac{A_{i+1}^{2}(x)}{A_{i}^{2}(x)+A_{i+1}^{2}(x)} \text {, then } A_{i}^{*}(x)+A_{i+1}^{*}(x)=1 \text {. } \\
& f_{\xi}(x)=\frac{y_{i+1}-y_{i}}{C}\left[A_{i}^{2}(x) \int_{0}^{A_{i}^{*}(x)}(1-t) d t+A_{i+1}^{2}(x) \int_{A_{i}^{*}(x)}^{1} t d t\right] \\
& =\frac{y_{i+1}-y_{i}}{2 C}\left[A_{i}^{2}(x) A_{i}^{*}(x)\left(1+A_{i+1}^{*}(x)\right)+A_{i+1}^{2}(x) A_{i+1}^{*}(x)\left(1+A_{i}^{*}(x)\right)\right] \\
& =\frac{1}{2 C}\left(y_{i+1}-y_{i}\right) \frac{A_{i}^{4}(x)+A_{i}^{2}(x) A_{i+1}^{2}(x)+A_{i+1}^{4}(x)}{A_{i}^{2}(x)+A_{i+1}^{2}(x)} \\
& =\frac{\left(1-A_{i}(x) A_{i+1}(x)\right)\left(1-3 A_{i}(x) A_{i+1}(x)\right)}{1-2 A_{i}(x) A_{i+1}(x)} \text {. }
\end{aligned}
$$

Because $(x, y) \in F_{i} \Leftrightarrow y \leq \frac{A_{i}^{2}(x) y_{i}+A_{i+1}^{2}(x) y_{i+1}}{A_{i}^{2}(x)+A_{i+1}^{2}(x)} \Leftrightarrow B_{i+1}(y) \leq \frac{A_{i}^{2}(x)}{A_{i}^{2}(x)+A_{i+1}^{2}(x)}$ $\Leftrightarrow \frac{A_{i+1}^{2}(x)}{A_{i}^{2}(x)} \leq \frac{B_{i}(y)}{B_{i+1}(y)} \Leftrightarrow \frac{A_{i+1}(x)}{1-A_{i+1}(x)} \leq \frac{\sqrt{B_{i}(y)}}{\sqrt{B_{i+1}(y)}} \Leftrightarrow A_{i+1}(x) \leq$ $\frac{\sqrt{B_{i}(y)}}{\sqrt{B_{i}(y)}+\sqrt{B_{i+1}(y)}} \Leftrightarrow x \leq \frac{\sqrt{B_{i}(y)}}{\sqrt{B_{i}(y)}+\sqrt{B_{i+1}(y)}} x_{i+1}+\frac{\sqrt{B_{i+1}(y)}}{\sqrt{B_{i}(y)}+\sqrt{B_{i+1}(y)}} x_{i} \leq$ $\tilde{x}_{i}$.

$$
\text { Let } \begin{aligned}
\widetilde{B}_{i}(y)= & \frac{\sqrt{B_{i}(y)}}{\sqrt{B_{i}(y)}+\sqrt{B_{i+1}(y)}} \text {, then } \\
f_{\eta}(y) & =\frac{1}{C}\left[\int_{x_{i}}^{\widetilde{x}_{i}} A_{i}^{2}(x) B_{i}(y) d x+\int_{\widetilde{x}_{i}}^{x_{i+1}} A_{i+1}^{2}(x) B_{i+1}(y) d x\right] \\
& =\frac{1}{C}\left[B_{i}(y) \int_{x_{i}}^{\widetilde{x}_{i}} A_{i}^{2}(x) d x+B_{i+1}(y) \int_{\widetilde{x}_{i}}^{x_{i+1}} A_{i+1}^{2}(x) d x\right] .
\end{aligned}
$$


Let $A_{i+1}(x)=t$, then $\widetilde{x}_{i}-x_{i}=\widetilde{B}_{i}(y)\left(x_{i+1}-x_{i}\right)$. So

$$
\begin{aligned}
f_{\eta}(y) & =\frac{x_{i+1}-x_{i}}{C}\left[B_{i}(y) \int_{0}^{\widetilde{B}_{i}(y)}(1-t)^{2} d t+B_{i+1}(y) \int_{\widetilde{B}_{i}(y)}^{1} t^{2} d t\right] \\
& =\frac{x_{i+1}-x_{i}}{3 C}\left\{B_{i}(y)\left[1-\widetilde{B}_{i+1}^{3}(y)\right]+B_{i+1}(y)\left[1-\widetilde{B}_{i+1}^{3}(y)\right]\right\} \\
& =\frac{1}{3 C}\left(x_{i+1}-x_{i}\right)\left(1-\frac{B_{i}(y) B_{i+1}(y)}{1+2 \sqrt{B_{i}(y) B_{i+1}(y)}}\right)
\end{aligned}
$$

Notes 1: In fact,Theorem 1 and Theorem 2 establish a method to construct probability distribution from the known data structures.

\section{The Numerical Characteristic of Two Distributions}

This section studies the numerical characteristic of two distributions described in Sect. 3, including the mathematical expectation, variance and covariance. We have the following results.

Theorem 3 Suppose $(\xi, \eta)$ obey bounded plot distribution, then

$$
\begin{aligned}
& \text { (1) } E(\xi)=\sum_{i=1}^{n-1} \omega_{i} \bar{x}_{i}, \quad E(\eta)=\sum_{i=1}^{n-1} \omega_{i} \bar{y}_{i}, \\
& (2) D(\xi) \approx \sum_{i=1}^{n-1} \omega_{i} x_{i} x_{i+1}-\left(\sum_{i=1}^{n-1} \omega_{i} \bar{x}_{i}\right), \quad D(\eta) \approx \sum_{i=1}^{n-1} \omega_{i} y_{i} y_{i+1}-\left(\sum_{i=1}^{n-1} \omega_{i} \bar{y}_{i}\right)^{2}, \\
& \text { (3) } \operatorname{Cov}(\xi, \eta) \approx \sum_{i=1}^{n-1} \omega_{i} \bar{z}_{i}-\left(\sum_{i=1}^{n-1} \omega_{i} \bar{x}_{i}\right)\left(\sum_{i=1}^{n-1} \omega_{i} \bar{y}_{i}\right),
\end{aligned}
$$

where

$$
\begin{aligned}
\omega_{i} & =\frac{\left(y_{i+1}-y_{i}\right)\left(x_{i+1}-x_{i}\right)}{\sum_{k=1}^{n-1}\left(y_{k+1}-y_{k}\right)\left(x_{k+1}-x_{k}\right)}, \quad \bar{x}_{i}=\frac{1}{2}\left(x_{i+1}+x_{i}\right), \quad \bar{y}_{i}=\frac{1}{2}\left(y_{i+1}+y_{i}\right), \\
z_{i} & =\frac{3}{5}\left(\frac{x_{i+1} y_{i+1}+x_{i} y_{i}}{2}\right)+\frac{2}{5}\left(\frac{x_{i+1} y_{i}+x_{i} y_{i+1}}{2}\right) .
\end{aligned}
$$


Proof (1) $E(\xi)=\frac{1}{2 T} \sum_{i=1}^{n-1}\left(y_{i+1}-y_{i}\right) \int_{x_{i}}^{x_{i+1}} x\left(1-2 A_{i}(x) A_{i+1}(x)\right) d x$

$$
\begin{aligned}
\int_{x_{i}}^{x_{i+1}} x\left(1-2 A_{i}(x) A_{i+1}(x)\right) d x= & \left(x_{i+1}-x_{i}\right) \int_{0}^{1}\left[x_{i}+\left(x_{i+1}-x_{i}\right) t\right]\left(1-2 t+2 t^{2}\right) d t \\
= & \left(x_{i+1}-x_{i}\right)\left[x_{i} \int_{0}^{1}\left(1-2 t+2 t^{2}\right) d t\right. \\
& \left.+\left(x_{i+1}-x_{i}\right) \int_{0}^{1}\left(1-2 t^{2}+2 t^{3}\right) d t\right] \\
= & \frac{1}{3}\left(x_{i+1}-x_{i}\right)\left(x_{i+1}+x_{i}\right) .
\end{aligned}
$$

Then

$$
\begin{aligned}
E(\xi) & =\frac{1}{2 T} \cdot \frac{1}{3} \sum_{i=1}^{n-1}\left(y_{i+1}-y_{i}\right)\left(x_{i+1}-x_{i}\right)\left(x_{i+1}+x_{i}\right) \\
& =\sum_{i=1}^{n-1} \frac{\left(y_{i+1}-y_{i}\right)\left(x_{i+1}-x_{i}\right)}{\sum_{k=1}^{n-1}\left(y_{k+1}-y_{k}\right)\left(x_{k+1}-x_{k}\right)} \cdot \frac{\left(x_{i+1}+x_{i}\right)}{2}=\sum_{i=1}^{n-1} \omega_{i} \bar{x}_{i} .
\end{aligned}
$$

Similarly,

$$
E(\eta)=\sum_{i=1}^{n-1} \omega_{i} \bar{y}_{i}
$$

(2) $E\left(\xi^{2}\right)=\frac{1}{2 T} \sum_{i=1}^{n-1}\left(y_{i+1}-y_{i}\right) \int_{x_{i}}^{x_{i+1}} x^{2}\left(1-2 A_{i}(x) A_{i+1}(x)\right) d x$

$$
\begin{aligned}
\int_{x_{i}}^{x_{i+1}} x^{2}\left(1-2 A_{i}(x) A_{i+1}(x)\right) d x= & \left(x_{i+1}-x_{i}\right) \int_{0}^{1}\left[x_{i}+\left(x_{i+1}-x_{i}\right) t\right]^{2}\left(1-2 t+2 t^{2}\right) d t \\
= & \left(x_{i+1}-x_{i}\right) \int_{0}^{1}\left[x_{i}^{2}+2 x_{i}\left(x_{i+1}-x_{i}\right) t\right. \\
& \left.+\left(x_{i+1}-x_{i}\right)^{2} t^{2}\right]\left(1-2 t+2 t^{2}\right) d t \\
= & \left(x_{i+1}-x_{i}\right)\left[x_{i}^{2} \int_{0}^{1}\left(1-2 t+2 t^{2}\right) d t\right. \\
& +2 x_{i}\left(x_{i+1}-x_{i}\right) \int_{0}^{1}\left(t-2 t^{2}+2 t^{3}\right) d t \\
& \left.+\left(x_{i+1}-x_{i}\right)^{2} \int_{0}^{1}\left(t^{2}-2 t^{3}+2 t^{4}\right) d t\right] \\
= & \frac{2}{3}\left(x_{i+1}-x_{i}\right) x_{i} x_{i+1}+\frac{7}{30}\left(x_{i+1}-x_{i}\right)^{3} .
\end{aligned}
$$


Then

$$
\begin{aligned}
E\left(\xi^{2}\right)= & \frac{1}{2 T} \cdot \frac{2}{3} \sum_{i=1}^{n-1}\left(y_{i+1}-y_{i}\right)\left(x_{i+1}-x_{i}\right) x_{i} x_{i+1} \\
& +\frac{17}{30} \cdot \frac{1}{2 T} \sum_{i=1}^{n-1}\left(y_{i+1}-y_{i}\right)\left(x_{i+1}-x_{i}\right)^{3} \\
= & \sum_{i=1}^{n-1} \frac{\left(y_{i+1}-y_{i}\right)\left(x_{i+1}-x_{i}\right)}{\sum_{k=1}^{n-1}\left(y_{k+1}-y_{k}\right)\left(x_{k+1}-x_{k}\right)} x_{i} x_{i+1} \\
& +\frac{17}{20} \sum_{i=1}^{n-1} \frac{\left(y_{i+1}-y_{i}\right)\left(x_{i+1}-x_{i}\right)}{\sum_{k=1}^{n-1}\left(y_{k+1}-y_{k}\right)\left(x_{k+1}-x_{k}\right)}\left(x_{i+1}-x_{i}\right)^{2} \\
= & \sum_{i=1}^{n-1} \omega_{i} x_{i} x_{i+1}+\frac{17}{20} \sum_{i=1}^{n-1} \omega_{i}\left(x_{i+1}-x_{i}\right)^{2} .
\end{aligned}
$$

Let $\Delta_{n}=\max _{1 \leq i \leq n-1}\left(x_{i+1}-x_{i}\right)^{2}$. Then

$$
\frac{17}{20} \sum_{i=1}^{n-1} \omega_{i}\left(x_{i+1}-x_{i}\right)^{2} \leq \frac{17}{20} \Delta_{n}
$$

When $n$ is sufficiently large, and $\Delta_{n}$ is sufficiently small,

$$
E\left(\xi^{2}\right) \approx \sum_{i=1}^{n-1} \omega_{i} x_{i} x_{i+1}
$$

Then

$$
D(\xi) \approx \sum_{i=1}^{n-1} \omega_{i} x_{i} x_{i+1}-\left(\sum_{i=1}^{n-1} \omega_{i} \bar{x}_{i}\right)^{2}
$$

In a similar way, $E\left(\eta^{2}\right)=\sum_{i=1}^{n-1} \omega_{i} y_{i} y_{i+1}+\frac{17}{20} \sum_{i=1}^{n-1} \omega_{i}\left(y_{i+1}-y_{i}\right)^{2} \approx$ $\sum_{i=1}^{n-1} \omega_{i} y_{i} y_{i+1}$.

Then $D(\eta) \approx \sum_{i=1}^{n-1} \omega_{i} y_{i} y_{i+1}-\left(\sum_{i=1}^{n-1} \omega_{i} \bar{y}_{i}\right)^{2}$. 


$$
\begin{aligned}
E(\xi \eta)= & \sum_{i=1}^{n-1} \int_{I_{i} \times J_{i}} x y f(x, y) d y d x=\sum_{i=1}^{n-1} \int_{x_{i}}^{x_{i+1}} x \int_{y_{i}}^{y_{i+1}} y_{i} f(x, y) d y d x \\
= & \sum_{i=1}^{n-1} \frac{1}{T} \int_{x_{i}}^{x_{i+1}} x\left[\int_{y_{i}}^{y_{i}^{*}} y\left(A_{i}(x)+B_{i}(y)-1\right) d y+\int_{y_{i}^{*}}^{y_{i+1}} y\left(A_{i+1}(x)\right.\right. \\
& \left.\left.+B_{i+1}(y)-1\right) d y\right] d x \\
& \int_{y_{i}}^{y_{i}^{*}} y\left(A_{i}(x)+B_{i}(y)-1\right) d y=\left(y_{i+1}-y_{i}\right) \\
& \times \int_{0}^{A_{i}(x)}\left[y_{i}+\left(y_{i+1}-y_{i}\right) t\right]\left[A_{i}(x)-1+1-t\right] d t \\
= & \left(y_{i+1}-y_{i}\right)\left[A_{i}(x) \int_{0}^{A_{i}(x)}\left(y_{i}+\left(y_{i+1}-y_{i}\right) t\right) d t\right. \\
& \left.-\int_{0}^{A_{i}(x)}\left(y_{i} t+\left(y_{i+1}-y_{i}\right) t^{2}\right) d t\right] \\
& \left.+\int_{A_{i}(x)}^{1}\left(y_{i+1}-y_{i}\right)\left(t^{2}-A_{i}(x)\right) d t\right] \\
= & \left(y_{i+1}-y_{i}\right)\left[\frac{1}{3} y_{i+1}+\frac{1}{6} y_{i}+\frac{1}{2} y_{i} A_{i}^{2}(x)\right. \\
& \int_{y_{i}^{*}}^{y_{i+1}} y\left(y_{i}\left(A_{i}(x)+B_{i} A_{i}^{2}(x)+\frac{1}{6}\left(y_{i+1}-y_{i}\right) A_{i}^{3}(x)\right]\right. \\
& \times \int_{A_{i}(x)}^{1}\left[y_{i}+\left(y_{i}\right) A_{i}(x)+\frac{1}{6}\left(y_{i+1}-y_{i}\right) A_{i}^{3}(x)\right] \\
= & \left(y_{i+1}-y_{i}\right)\left[\int_{A_{i}(x)}^{1} y_{i} t d t-y_{i} A_{i}(x)\left(1-A_{i}(x)\right)\right. \\
& \\
& \\
& \\
&
\end{aligned}
$$

Then

$$
\begin{aligned}
E(\xi \eta)= & \frac{1}{T} \sum_{i=1}^{n-1}\left(y_{i+1}-y_{i}\right) \int_{x_{i}}^{x_{i+1}} x\left[\frac{1}{2} y_{i} A_{i}^{2}(x)+\frac{1}{6}\left(y_{i+1}-y_{i}\right) A_{i}^{3}(x)+\frac{1}{3} y_{i+1}+\frac{1}{6} y_{i}\right. \\
& \left.+\frac{1}{2} y_{i} A_{i}^{2}(x)-\frac{1}{2}\left(y_{i+1}+y_{i}\right) A_{i}(x)+\frac{1}{6}\left(y_{i+1}-y_{i}\right) A_{i}^{3}(x)\right] \\
= & \frac{1}{T} \sum_{i=1}^{n-1}\left(y_{i+1}-y_{i}\right) \int_{x_{i}}^{x_{i+1}} x\left[y_{i} A_{i}^{2}(x)+\frac{1}{3}\left(y_{i+1}-y_{i}\right) A_{i}^{3}(x)\right.
\end{aligned}
$$




$$
\left.-\frac{1}{2}\left(y_{i+1}+y_{i}\right) A_{i}(x)+\frac{1}{3} y_{i+1}+\frac{1}{6} y_{i}\right] d x .
$$

Let $A_{i}(x)=\frac{x_{i+1}-x}{x_{i+1}-x_{i}}=t$. Then $d x=-\left(x_{i+1}-x_{i}\right) d t$. Then

$$
\begin{aligned}
E(\xi \eta)= & \frac{1}{T} \sum_{i=1}^{n-1}\left(y_{i+1}-y_{i}\right)\left(x_{i+1}-x_{i}\right) \int_{0}^{1}\left[x_{i+1}-\left(x_{i+1}-x_{i}\right) t\right]\left[y_{i} t^{2}+\frac{1}{3}\left(y_{i+1}-y_{i}\right) t^{3}\right. \\
& \left.-\frac{1}{2}\left(y_{i+1}+y_{i}\right) t+\frac{1}{3} y_{i+1}+\frac{1}{6} y_{i}\right] d t \\
= & 3 \sum_{i=1}^{n-1} \omega_{i}\left[x _ { i + 1 } \int _ { 0 } ^ { 1 } \left(y_{i} t^{2}+\frac{1}{3}\left(y_{i+1}-y_{i}\right) t^{3}-\frac{1}{2}\left(y_{i+1}+y_{i}\right) t+\frac{1}{3} y_{i+1}\right.\right. \\
& \left.+\frac{1}{6} y_{i}\right) d t-\left(x_{i+1}-x_{i}\right) \int_{0}^{1}\left(y_{i} t^{3}+\frac{1}{3}\left(y_{i+1}-y_{i}\right) t^{4}-\frac{1}{2}\left(y_{i+1}+y_{i}\right) t^{2}+\left(\frac{1}{3} y_{i+1}\right.\right. \\
& \left.\left.\left.+\frac{1}{6} y_{i}\right) t\right) d t\right] \\
= & 3 \sum_{i=1}^{n-1} \omega_{i}\left[x _ { i + 1 } \left(\frac{1}{3} y_{i}+\frac{1}{12}\left(y_{i+1}-y_{i}\right)-\frac{1}{4}\left(y_{i+1}+y_{i}\right)\right.\right. \\
& \left.+\frac{1}{3} y_{i+1}+\frac{1}{6} y_{i}\right)-\left(x_{i+1}-x_{i}\right)\left(\frac{1}{4} y_{i}+\frac{1}{15}\left(y_{i+1}-y_{i}\right)-\frac{1}{6}\left(y_{i+1}+y_{i}\right)\right. \\
& \left.\left.+\frac{1}{6} y_{i+1}+\frac{1}{12} y_{i}\right)\right] \\
= & 3 \sum_{i=1}^{n-1} \omega_{i}\left[\frac{1}{10}\left(x_{i+1} y_{i+1}+x_{i} y_{i}\right)+\frac{1}{15}\left(x_{i+1} y_{i}+x_{i} y_{i+1}\right)\right] \\
= & \sum_{i=1}^{n-1} \omega_{i}\left[\left(\frac{x_{i+1} y_{i+1}+x_{i} y_{i}}{2}\right)-\frac{2}{5}\left(x_{i+1}-x_{i}\right)\left(y_{i+1}-y_{i}\right)\right] \\
& \sum_{i=1}^{n-1} \omega_{i} \bar{z}_{i}-\frac{2}{5} \sum_{i=1}^{n-1} \omega_{i}\left(x_{i+1}-x_{i}\right)\left(y_{i+1}-y_{i}\right) . \\
& \\
&
\end{aligned}
$$

Then $\operatorname{Cov}(\xi, \eta)=\mathrm{E}(\xi \eta)-\mathrm{E}(\xi) \mathrm{E}(\eta) \approx \sum_{\mathrm{i}=1}^{\mathrm{n}-1} \omega_{\mathrm{i}} \overline{\mathrm{z}}_{\mathrm{i}}-\left(\sum_{\mathrm{i}=1}^{\mathrm{n}-1} \omega_{\mathrm{i}} \overline{\mathrm{x}}_{\mathrm{i}}\right)\left(\sum_{\mathrm{i}=1}^{\mathrm{n}-1} \omega_{\mathrm{i}} \overline{\mathrm{y}}_{\mathrm{i}}\right)$.

Theorem 4 Suppose $(\xi, \eta)$ obey Larsen square distribution, then:

$$
\begin{aligned}
& \text { (1) } E(\xi)=\sum_{i=1}^{n-1} \omega_{i} \bar{x}_{i}, E(\eta)=\sum_{i=1}^{n-1} \omega_{i} \bar{y}_{i} . \\
& \text { (2) } D(\xi) \approx \sum_{i=1}^{n-1} \omega_{i} x_{i} x_{i+1}-\left(\sum_{i=1}^{n-1} \omega_{i} \bar{x}_{i}\right)^{2}, D(\eta) \approx \sum_{i=1}^{n-1} \omega_{i} y_{i} y_{i+1}-\left(\sum_{i=1}^{n-1} \omega_{i} \bar{y}_{i}\right)^{2}
\end{aligned}
$$


(3) $\operatorname{Cov}(\xi, \eta) \approx \sum_{i=1}^{n-1} \omega_{i} \bar{z}_{i}-\left(\sum_{i=1}^{n-1} \omega_{i} \bar{x}_{i}\right)\left(\sum_{i=1}^{n-1} \omega_{i} \bar{y}_{i}\right)$,

where $\bar{x}_{i}=\frac{1}{2}\left(x_{i}+x_{i+1}\right), \bar{y}_{i}=\frac{1}{2}\left(y_{i}+y_{i+1}\right), \bar{z}_{i}=\frac{1}{2}\left(x_{i} y_{i}+x_{i+1} y_{i+1}\right)$.

Proof

$$
\begin{aligned}
E(\xi) & =\frac{1}{2 C} \sum_{i=1}^{n-1}\left(y_{i+1}-y_{i}\right) \int_{x_{i}}^{x_{i+1}} x \frac{\left(1-A_{i}(x) A_{i+1}(x)\right)\left(1-3 A_{i}(x) A_{i+1}(x)\right)}{1-2 A_{i}(x) A_{i+1}(x)} d x \\
& =\frac{1}{2 C} \sum_{i=1}^{n-1}\left(x_{i+1}-x_{i}\right)\left(y_{i+1}-y_{i}\right) \int_{0}^{1}\left[x_{i}+\left(x_{i+1}-x_{i}\right) t\right] \frac{\left(1-t+t^{2}\right)\left(1-3 t+3 t^{2}\right)}{\left(1-2 t+2 t^{2}\right)} d t \\
& =\frac{1}{2 C} \sum_{i=1}^{n-1}\left(x_{i+1}-x_{i}\right)\left(y_{i+1}-y_{i}\right)\left(\frac{1}{2}-\frac{\pi}{16}\right)\left(x_{i+1}+x_{i}\right) \\
& =\sum_{i=1}^{n-1} \frac{\left(x_{i+1}-x_{i}\right)\left(y_{i+1}-y_{i}\right)}{\sum_{k=1}^{n-1}\left(x_{k+1}-x_{k}\right)\left(y_{k+1}-y_{k}\right)} \frac{x_{i+1}+x_{i}}{2}=\sum_{i=1}^{n-1} \omega_{i} \bar{x}_{i} \\
E(\eta) & =\frac{1}{3 C} \sum_{i=1}^{n-1}\left(x_{i+1}-x_{i}\right) \int_{y_{i}}^{y_{i+1}} y\left(1-\frac{B_{i}(y) B_{i+1}(y)}{1+2 \sqrt{B_{i}(y) B_{i+1}(y)}}\right) d y \\
& =\frac{1}{3 C} \sum_{i=1}^{n-1}\left(x_{i+1}-x_{i}\right)\left(y_{i+1}-y_{i}\right) \int_{0}^{1}\left[y_{i}+\left(y_{i+1}-y_{i}\right) t\right]\left[1-\frac{\left(t-t^{2}\right)}{1+2 \sqrt{\left(t-t^{2}\right)}}\right] d t \\
& =\frac{1}{3 C} \sum_{i=1}^{n-1}\left(x_{i+1}-x_{i}\right)\left(y_{i+1}-y_{i}\right)\left(\frac{3}{4}-\frac{2}{32} \pi\right)\left(y_{i+1}+y_{i}\right) \\
& =\sum_{i=1}^{n-1} \frac{\left(x_{i+1}-x_{i}\right)\left(y_{i+1}-y_{i}\right)}{\sum_{k=1}^{n-1}\left(x_{k+1}-x_{k}\right)\left(y_{k+1}-y_{k}\right)} \frac{y_{i+1}+y_{i}}{2}=\sum_{i=1}^{n-1} \omega_{i} \bar{y}_{i} \\
& \\
& =1 \\
&
\end{aligned}
$$

(2)Let $k=\frac{1}{2}-\frac{1}{16} \pi$, then

$$
\begin{aligned}
E\left(\xi^{2}\right)= & \frac{1}{2 C} \sum_{i=1}^{n-1}\left(x_{i+1}-x_{i}\right)\left(y_{i+1}-y_{i}\right) \\
& \times \int_{0}^{1}\left[x_{i}+\left(x_{i+1}-x_{i}\right) t\right]^{2} \frac{\left(1-t+t^{2}\right)\left(1-3 t+3 t^{2}\right)}{\left(1-2 t+2 t^{2}\right)} d t \\
= & \frac{1}{2 C} \sum_{i=1}^{n-1}\left(x_{i+1}-x_{i}\right)\left(y_{i+1}-y_{i}\right)\left(-\frac{1}{8} x_{i} x_{i+1} \pi+\frac{17}{30} x_{i} x_{i+1}+\frac{13}{60} x_{i}^{2}+\frac{13}{60} x_{i+1}^{2}\right)
\end{aligned}
$$




$$
\begin{aligned}
= & \frac{1}{2 C} \sum_{i=1}^{n-1} \frac{\left(x_{i+1}-x_{i}\right)\left(y_{i+1}-y_{i}\right)}{\sum_{k=1}^{n-1}\left(x_{k+1}-x_{k}\right)\left(y_{k+1}-y_{k}\right)}\left[2\left[\frac{1}{2}-\frac{1}{16} \pi\right] x_{i} x_{i+1}\right. \\
& \left.+\frac{13}{60}\left(x_{i}^{2}+x_{i+1}^{2}-2 x_{i} x_{i+1}\right)\right] \\
= & \sum_{i=1}^{n-1} \omega_{i} x_{i} x_{i+1}+\frac{13}{120 k} \sum_{i=1}^{n-1} \omega_{i}\left(x_{i+1}-x_{i}\right)^{2} \approx \sum_{i=1}^{n-1} \omega_{i} x_{i} x_{i+1} .
\end{aligned}
$$

So, $D(\xi)=E\left(\xi^{2}\right)-(E(\xi))^{2} \approx \sum_{i=1}^{n-1} \omega_{i} x_{i} x_{i+1}-\left(\sum_{i=1}^{n-1} \omega_{i} \bar{x}_{i}\right)^{2}$

$$
\begin{aligned}
E\left(\eta^{2}\right)= & \frac{1}{3 C} \sum_{i=1}^{n-1}\left(x_{i+1}-x_{i}\right)\left(y_{i+1}-y_{i}\right) \int_{0}^{1}\left[y_{i}+\left(y_{i+1}-y_{i}\right) t\right]^{2}\left[1-\frac{t-t^{2}}{1+2 \sqrt{t-t^{2}}}\right] d t \\
= & \frac{1}{3 C} \sum_{i=1}^{n-1} \frac{\left(x_{i+1}-x_{i}\right)\left(y_{i+1}-y_{i}\right)}{\sum_{k=1}^{n-1}\left(x_{k+1}-x_{k}\right)\left(y_{k+1}-y_{k}\right)}\left[3\left(\frac{1}{2}-\frac{1}{16} \pi\right) y_{i} y_{i+1}\right. \\
& \left.+3\left(\frac{5}{36}-\frac{3}{256} \pi\right)\left(y_{i+1}-y_{i}\right)^{2}\right] \\
= & \sum_{i=1}^{n-1} \omega_{i} y_{i} y_{i+1}+\left(\frac{5}{36}-\frac{3}{256} \pi\right) \frac{1}{k}\left(y_{i+1}-y_{i}\right)^{2} \\
\approx & \sum_{i=1}^{n-1} \omega_{i} y_{i} y_{i+1} .
\end{aligned}
$$

So $D(\eta)=E\left(\eta^{2}\right)-(E(\eta))^{2} \approx \sum_{i=1}^{n-1} \omega_{i} y_{i} y_{i+1}-\left(\sum_{i=1}^{n-1} \omega_{i} \bar{y}_{i}\right)^{2}$.

$$
\begin{aligned}
E(\xi \eta)= & \sum_{i=1}^{n-1} \int_{x_{i}}^{x_{i+1}} \int_{y_{i}}^{y_{i+1}} x y f(x, y) d y d x \\
= & \frac{1}{C} \sum_{i=1}^{n-1}\left[\int_{x_{i}}^{x_{i+1}} x \int_{y_{i}}^{\widetilde{y}_{i}} y A_{i}^{2}(x) B_{i}(y) d y d x\right. \\
& \left.+\int_{x_{i}}^{x_{i+1}} x \int_{\tilde{y}_{i}}^{y_{i+1}} y A_{i+1}^{2}(x) B_{i+1}(y) d y d x\right] \\
\triangle_{1}= & \int_{x_{i}}^{x_{i+1}} x \int_{y_{i}}^{\tilde{y}_{i}} y A_{i}^{2}(x) B_{i}(y) d y d x=\int_{x_{i}}^{x_{i+1}} x A_{i}^{2}(x) \int_{y_{i}}^{\tilde{y}_{i}} y B_{i}(y) d y d x
\end{aligned}
$$




$$
\begin{aligned}
= & \int_{x_{i}}^{x_{i+1}} x A_{i}^{2}(x)\left(y_{i+1}-y_{i}\right) \int_{0}^{A_{i}^{*}(x)}\left[y_{i}+\left(y_{i+1}-y_{i}\right) t\right](1-t) d t d x \\
= & \left(y_{i+1}-y_{i}\right) \int_{x_{i}}^{x_{i+1}}\left[x A _ { i } ^ { 2 } ( x ) \left[y_{i}\left(A_{i}^{*}(x)-\frac{1}{2}\left(A_{i}^{*}(x)\right)^{2}\right]+\left(y_{i+1}-y_{i}\right)\right.\right. \\
& \left.\times\left[\frac{1}{2}\left(A_{i}^{*}(x)\right)^{2}-\frac{1}{3}\left(A_{i}^{*}(x)\right)^{3}\right]\right] d x \\
= & \frac{7}{72} x_{i+1} y_{i}+\frac{19}{144} x_{i+1} y_{i+1}+\frac{1}{16} x_{i} y_{i+1}-\frac{1}{24} x_{i} y_{i}-\frac{\pi}{128}\left[x_{i} y_{i+1}+3 x_{i+1} y_{i}\right. \\
& \left.+5 x_{i+1} y_{i+1}-5 x_{i} y_{i}\right] . \\
\triangle_{2}= & \int_{x_{i}}^{x_{i+1}} x \int_{\tilde{y}_{i}}^{y_{i+1}} y A_{i+1}^{2}(x) B_{i+1}(y) d y d x \\
& =\int_{x_{i}}^{x_{i+1}} x A_{i+1}^{2}(x) \int_{\tilde{y}_{i}}^{y_{i+1}} y B_{i+1}(y) d y d x \\
= & \frac{7}{72} x_{i} y_{i+1}+\frac{19}{144} x_{i} y_{i}+\frac{1}{16} x_{i+1} y_{i}-\frac{1}{24} x_{i+1} y_{i+1}-\frac{\pi}{128}\left[x_{i+1} y_{i}+3 x_{i} y_{i+1}\right. \\
& \left.+5 x_{i} y_{i}-5 x_{i+1} y_{i+1}\right] . \\
= & \left(y_{i+1}-y_{i}\right) \int_{x_{i}}^{x_{i+1}} x A_{i+1}^{2}(x) \int_{A_{i}^{*}(x)}^{1}\left[y_{i}+\left(y_{i+1}-y_{i}\right) t\right] t d t d x \\
& +\frac{1}{3}\left(y_{i+1}-y_{x_{i}}^{x_{i+1}} x A_{i+1}^{2}(x)\left[\frac{1}{2} y_{i}\left(1-\left(A_{i}^{*}(x)\right)^{2}\right)\right.\right. \\
& \\
& \\
& \\
&
\end{aligned}
$$

Then

$$
\begin{aligned}
\triangle_{1}+\triangle_{2}= & \frac{1}{16}\left(x_{i+1} y_{i}+x_{i} y_{i+1}\right)+\frac{7}{72}\left(x_{i+1} y_{i}+x_{i} y_{i+1}\right)+\frac{9}{144}\left(x_{i} y_{i}+x_{i+1} y_{i+1}\right) \\
& -\frac{1}{24}\left(x_{i} y_{i}+x_{i+1} y_{i+1}\right)-\frac{\pi}{128}\left(4 x_{i} y_{i+1}+4 x_{i+1} y_{i}\right) \\
= & \left(\frac{1}{4}-\frac{\pi}{32}\right)\left(x_{i} y_{i}+x_{i+1} y_{i+1}\right)+\frac{23}{144}\left(y_{i+1}-y_{i}\right)\left(x_{i+1}-x_{i}\right) .
\end{aligned}
$$

Then

$$
\begin{aligned}
E(\xi \eta)= & \frac{1}{C} \sum_{i=1}^{n-1}\left(y_{i+1}-y_{i}\right)\left(x_{i+1}-x_{i}\right)\left[\left(\frac{1}{4}-\frac{\pi}{32}\right)\left(x_{i} y_{i}+x_{i+1} y_{i+1}\right)\right. \\
& \left.+\frac{23}{144}\left(y_{i+1}-y_{i}\right)\left(x_{i+1}-x_{i}\right)\right]
\end{aligned}
$$




$$
\begin{aligned}
& =\sum_{i=1}^{n-1} \omega_{i}\left[\frac{x_{i} y_{i}+x_{i+1} y_{i+1}}{2}\right]+\frac{23}{144 k}\left(y_{i+1}-y_{i}\right)\left(x_{i+1}-x_{i}\right) \\
& =\sum_{i=1}^{n-1} \omega_{i} \bar{z}_{i}+\frac{23}{144 k} \sum_{i=1}^{n-1} \omega_{i}\left(y_{i+1}-y_{i}\right)\left(x_{i+1}-x_{i}\right) \approx \sum_{i=1}^{n-1} \omega_{i} \bar{z}_{i} .
\end{aligned}
$$

$\operatorname{So} \operatorname{Cov}(\xi, \eta) \approx \sum_{i=1}^{n-1} \omega_{i} \bar{z}_{i}-\left(\sum_{i=1}^{n-1} \omega_{i} \bar{x}_{i}\right)\left(\sum_{i=1}^{n-1} \omega_{i} \bar{y}_{i}\right)$.

Notes 2: (1)From Theorem 3 and Theorem 4 we can see that, bounded integral distribution and Larsen square distribution have the same mathematical expectation and nearly the same variance and covariance.

(2) The above results are obtained in the suppose which is $a=x_{1}<x_{2}<\ldots<$ $x_{n}=b, c=y_{1}<y_{2}<\ldots<y_{n}=d$. And we can get the similar conclusion when $a=x_{1}<x_{2}<\ldots<x_{n}=b, c=y_{1}>y_{2}>\ldots>y_{n}=d$.

\section{Center-of-Gravity Fuzzy Trustworthiness System}

Let $f(x, y)$ be the probability density function obtained in our study, we can know that from the discussion above,

$$
\bar{S}(x)=\frac{\int_{c}^{d} y B^{*}(y) d y}{\int_{c}^{d} B^{*}(y) d y}=\frac{\int_{c}^{d} y R(x, y) d y}{\int_{c}^{d} R(x, y) d y}=\frac{\int_{-\infty}^{+\infty} y f(x, y) d y}{\int_{-\infty}^{+\infty} f(x, y) d y},
$$

so we have the following theorem.

Theorem 5 If $\theta=$ Bounded Product implication, where $\bar{S}_{T}(x)=A_{i}^{*}(x) y_{i}+$ $A_{i+1}^{*}(x) y_{i+1}$, where

$$
\begin{aligned}
A_{i}^{*}(x) & =\frac{\frac{2}{3} A_{i+1}^{3}(x)-A_{i+1}(x)+\frac{2}{3}}{1-2 A_{i}(x) A_{i+1}(x)} \\
A_{i+1}^{*}(x) & =1-A_{i}^{*}(x)=\frac{\frac{2}{3} A_{i}^{3}(x)-A_{i}(x)+\frac{2}{3}}{1-2 A_{i}(x) A_{i+1}(x)}
\end{aligned}
$$

and $\bar{S}_{T}\left(x_{i}\right)=\frac{2}{3} y_{i}+\frac{1}{3} y_{i+1}, \bar{S}_{L}\left(x_{i+1}\right)=\frac{1}{3} y_{i}+\frac{2}{3} y_{i+1}$.

Proof From the theorem above we can know that:

$$
\int_{-\infty}^{+\infty} f(x, y) d y=f_{\xi}(x)=\frac{y_{i+1}-y_{i}}{T}\left[\frac{1}{2}-A_{i}(x) A_{i+1}(x)\right]
$$


and

$$
\begin{aligned}
\int_{-\infty}^{+\infty} y f(x, y) d y= & \frac{1}{T}\left[\int_{y_{i}}^{y_{i}^{*}} y\left(A_{i}(x)+B_{i}(x)-1\right) d y\right. \\
& \left.+\int_{y_{i}^{*}}^{y_{i+1}} y\left(A_{i+1}(x)+B_{i+1}(x)-1\right) d y\right] \\
= & \frac{y_{i+1}-y_{i}}{T}\left[\left(A_{i}^{2}(x)-\frac{1}{3} A_{i}^{3}(x)-\frac{1}{2} A_{i}(x)+\frac{1}{6}\right) y_{i}\right. \\
& \left.+\left(\frac{1}{3} A_{i}^{3}(x)-\frac{1}{2} A_{i}(x)+\frac{1}{3}\right) y_{i+1}\right]
\end{aligned}
$$

so

$$
\begin{aligned}
\bar{S}_{T}(x)= & \frac{\int_{-\infty}^{+\infty} y f(x, y) d y}{\int_{-\infty}^{+\infty} f(x, y) d y}=\frac{\left(A_{i}^{2}(x)-\frac{1}{3} A_{i}^{3}(x)-\frac{1}{2} A_{i}(x)+\frac{1}{6}\right) y_{i}}{\frac{1}{2}-A_{i}(x) A_{i+1}(x)} \\
& +\frac{\left(\frac{1}{3} A_{i}^{3}(x)-\frac{1}{2} A_{i}(x)+\frac{1}{3}\right) y_{i+1}}{\frac{1}{2}-A_{i}(x) A_{i+1}(x)}=A_{i}^{*}(x) y_{i}+A_{i+1}^{*}(x) y_{i+1},
\end{aligned}
$$

and

$$
\begin{aligned}
A_{i}^{*}(x)+A_{i+1}^{*}(x)= & \frac{1}{\frac{1}{2}-A_{i}(x) A_{i+1}(x)}\left[A_{i}^{2}(x)-\frac{1}{3} A_{i}^{3}(x)-\frac{1}{2} A_{i}(x)\right. \\
& \left.+\frac{1}{6}+\frac{1}{3} A_{i}^{3}(x)-\frac{1}{2} A_{i}(x)+\frac{1}{3}\right]=\frac{\frac{1}{2}-A_{i}(x)\left(1-A_{i}(x)\right)}{\frac{1}{2}-A_{i}(x) A_{i+1}(x)}=1 .
\end{aligned}
$$

And because

$$
\begin{aligned}
& A_{i}^{2}(x)-\frac{1}{3} A_{i}^{3}(x)-\frac{1}{2} A_{i}(x)+\frac{1}{6}=\left(1-A_{i+1}(x)\right)^{2}-\frac{1}{3}\left(1-A_{i+1}(x)\right)^{3} \\
& -\frac{1}{2}\left(1-A_{i+1}(x)\right)+\frac{1}{6}=\frac{1}{3} A_{i+1}^{3}(x)-\frac{1}{2} A_{i+1}(x)+\frac{1}{3}
\end{aligned}
$$

we have

$$
A_{i}^{*}(x)=\frac{\frac{2}{3} A_{i+1}^{3}(x)-A_{i+1}(x)+\frac{2}{3}}{1-2 A_{i}(x) A_{i+1}(x)}, \quad A_{i+1}^{*}(x)=\frac{\frac{2}{3} A_{i}^{3}(x)-A_{i}(x)+\frac{2}{3}}{1-2 A_{i}(x) A_{i+1}(x)} .
$$


We study the fuzzy trustworthiness system exported by Larsen square distribution below.

Theorem $6 \bar{S}_{L^{2}}(x)=\frac{\int_{-\infty}^{+\infty} y f(x, y) d y}{\int_{-\infty}^{+\infty} f(x, y) d y}=C_{i}^{*}(x) y_{i}+C_{i+1}^{*}(x) y_{i+1}$, where

$$
C_{i+1}^{*}(x)=\frac{A_{i}^{6}(x)+2 A_{i+1}^{2}(x)\left(A_{i}^{2}(x)+A_{i+1}^{2}(x)\right)^{2}}{3\left(A_{i}^{4}(x)+A_{i}^{2}(x) A_{i+1}^{2}(x)+A_{i+1}^{2}(x)\right)\left(A_{i}^{2}(x)+A_{i+1}^{2}(x)\right)},
$$

$C_{i}^{*}(x) y_{i}+C_{i+1}^{*}(x) y_{i+1} \equiv 1$ and $\bar{S}_{L^{2}}\left(x_{i}\right)=\frac{2}{3} y_{i}+\frac{1}{3} y_{i+1}, \bar{S}_{L^{2}}\left(x_{i+1}\right)=\frac{1}{3} y_{i}+\frac{2}{3} y_{i+1}$.

Proof

$$
\begin{aligned}
& \int_{-\infty}^{+\infty} f(x, y) d y=\int_{y_{i}}^{y_{i+1}} f(x, y) d y \\
& =\frac{y_{i+1}-y_{i}}{2 C} \frac{\left(1-A_{i}(x) A_{i+1}(x)\right)\left(1-3 A_{i}(x) A_{i+1}(x)\right)}{1-2 A_{i}(x) A_{i+1}(x)} \\
& \int_{-\infty}^{+\infty} y f(x, y) d y=\frac{1}{C}\left[\int_{y_{i}}^{\widehat{y}_{i}} y A_{i}^{2}(x) B_{i}(y) d y+\int_{\widehat{y}_{i}}^{y_{i+1}} y A_{i+1}^{2}(x) B_{i+1}(y) d y\right] \\
& =\frac{y_{i+1}-y_{i}}{C}\left[\left[A_{i}^{2}(x)\left(A_{i}^{*}(x)-\left(A_{i}^{*}(x)\right)^{2}+\frac{1}{3}\left(A_{i}^{*}(x)\right)^{3}\right)\right.\right. \\
& \left.\left.\quad+A_{i+1}^{2}(x)\left(\frac{1}{2}\left(1-A_{i}^{*}(x)\right)^{2}\right)-\frac{1}{3}\left(1-\left(A_{i}^{*}(x)\right)^{3}\right)\right)\right] y_{i} \\
& \left.\quad+\left[A_{i}^{2}(x)\left(\frac{1}{2}\left(A_{i}^{*}(x)\right)^{2}-\frac{1}{3}\left(A_{i}^{*}(x)\right)^{3}\right)+\frac{1}{3} A_{i+1}^{2}(x)\left(1-\left(A_{i}^{*}(x)\right)^{3}\right)\right] y_{i+1}\right] \\
& \quad=\frac{y_{i+1}-y_{i}}{C}\left[D_{i}(x) y_{i}+D_{i+1}(x) y_{i+1}\right] .
\end{aligned}
$$

Then

$$
\begin{aligned}
D_{i}(x)+D_{i+1}(x) & =A_{i}^{2}(x)\left[A_{i}^{*}(x)-\frac{1}{2}\left(A_{i}^{*}(x)\right)^{2}\right]+\frac{1}{2} A_{i+1}^{2}(x)\left[1-\left(A_{i}^{*}(x)\right)^{2}\right] \\
& =A_{i}^{2}(x) A_{i}^{*}(x)+\frac{1}{2} A_{i+1}^{2}(x)-\frac{1}{2}\left(A_{i}^{*}(x)\right)^{2}\left(A_{i}^{2}(x)+A_{i+1}^{2}(x)\right) \\
& =\frac{2 A_{i}^{4}(x)+A_{i+1}^{2}(x)\left(A_{i}^{2}(x)+A_{i+1}^{2}(x)\right)-A_{i}^{4}(x)}{2\left(A_{i}^{2}(x)+A_{i+1}^{2}(x)\right)} \\
& =\frac{\left(1-A_{i}(x) A_{i+1}(x)\right)\left(1-3 A_{i}(x) A_{i+1}(x)\right)}{2\left(1-2 A_{i}(x) A_{i+1}(x)\right)}
\end{aligned}
$$


So, $C_{i}^{*}(x)+C_{i+1}^{*}(x)=1$. And

$$
\begin{aligned}
D_{i+1}(x) & =A_{i}^{2}(x)\left[\frac{1}{2}\left(A_{i}^{*}(x)\right)^{2}-\frac{1}{3}\left(A_{i}^{*}(x)\right)^{3}\right]+\frac{1}{3} A_{i+1}^{2}(x)\left(1-\left(A_{i}^{*}(x)\right)^{3}\right) \\
& =\frac{3 A_{i}^{2}(x) A_{i}^{4}(x)\left(A_{i}^{2}(x)+A_{i+1}^{2}(x)\right)-2 A_{i}^{8}(x)+2 A_{i+1}^{2}(x)\left(\left(A_{i}^{2}(x)+A_{i+1}^{2}(x)\right)^{3}-A_{i}^{6}(x)\right)}{6\left(A_{i}^{2}(x)+A_{i+1}^{2}(x)\right)^{3}} \\
& =\frac{A_{i}^{8}(x)+3 A_{i}^{6}(x) A_{i+1}^{2}(x)+2 A_{i+1}^{8}(x)+6 A_{i}^{4}(x) A_{i+1}^{4}(x)+6 A_{i}^{2}(x) A_{i+1}^{2}(x)}{6\left(A_{i}^{2}(x)+A_{i+1}^{2}(x)\right)^{3}} \\
& =\frac{A_{i}^{6}(x)+2 A_{i+1}^{2}(x)\left(A_{i}^{2}(x)+A_{i+1}^{2}(x)\right)^{2}}{6\left(A_{i}^{2}(x)+A_{i+1}^{2}(x)\right)^{2}},
\end{aligned}
$$

thus

$$
\begin{aligned}
C_{i+1}^{*}(x) & =\frac{2 D_{i+1}(x)}{\frac{A_{i}^{4}(x)+A_{i}^{2}(x) A_{i+1}^{2}(x)+A_{i+1}^{4}(x)}{\left(A_{i+1}^{2}(x)+A_{i}^{2}(x)\right)}} \\
& =\frac{A_{i}^{6}(x)+2 A_{i+1}^{2}(x)\left(A_{i}^{2}(x)+A_{i+1}^{2}(x)\right)^{2}}{3\left(A_{i}^{4}(x)+A_{i}^{2}(x) A_{i+1}^{2}(x)+A_{i+1}^{4}(x)\right)\left(A_{i}^{2}(x)+A_{i+1}^{2}(x)\right)} \\
& =\frac{A_{i}^{6}(x)+2 A_{i+1}^{2}(x)\left(A_{i}^{2}(x)+A_{i+1}^{2}(x)\right)^{2}}{3\left(1-A_{i}(x) A_{i+1}(x)\right)\left(1-2 A_{i}(x) A_{i+1}(x)\right)\left(1-3 A_{i}(x) A_{i+1}(x)\right)} .
\end{aligned}
$$

So $\bar{S}(x)=C_{i}^{*}(x) y_{i}+C_{i+1}^{*}(x) y_{i+1}, C_{i+1}^{*}\left(x_{i+1}\right)=\frac{2}{3} ; C_{i}^{*}\left(x_{i+1}\right)=1-\frac{2}{3}=$ $\frac{1}{3} ; C_{i+1}^{*}\left(x_{i}\right)=\frac{1}{3} ; C_{i}^{*}\left(x_{i+1}\right)=1-\frac{1}{3}=\frac{2}{3}$, so $\bar{S}\left(x_{i}\right)=\frac{2}{3} y_{i}+\frac{1}{3} y_{i+1}, \bar{S}\left(x_{i+1}\right)=$ $\frac{1}{3} y_{i}+\frac{2}{3} y_{i+1}$.

We study the universal approximations of fuzzy trustworthiness systems $\bar{S}_{T}(x)$ and $\bar{S}_{L^{2}}(x)$. Suppose $s(x)$ is a known system and $s\left(x_{i}\right)=y_{i}$. Let $h=$ $\max _{1 \leq i \leq n-1} \Delta x_{i},\|s\|_{\infty}=\max _{x \in[a, b]}|s(x)|$. Suppose $F_{1}(x)=A_{i}(x) y_{i}+A_{i+1}(x) y_{i+1},(x \in$ $\left.\left[x_{i}, x_{i+1}\right]\right)$. From reference [11], we can know that: $\left\|s-F_{1}\right\| \leq \frac{1}{8}\left\|s^{\prime \prime}\right\|_{\infty} h^{2}$. So we have

Theorem 7 When $\bar{S}(x) \in\left\{\bar{S}_{T}(x), \bar{S}_{L^{2}}(x)\right\}$,

$$
\left\|\bar{S}(x)-F_{1}(x)\right\| \leq \frac{1}{3}\left\|s^{\prime}\right\|_{\infty} h .
$$

Proof (1) When $\bar{S}(x)=\bar{S}_{T}(x)$,

$$
A_{i}^{*}(x)=\frac{\frac{1}{3} A_{i+1}^{3}(x)-A_{i+1}(x)+\frac{2}{3}}{1-2 A_{i}(x) A_{i+1}(x)} .
$$


Then

$$
\begin{aligned}
A_{i}^{*}(x)-A_{i}(x) & =\frac{\frac{1}{3} A_{i+1}^{3}(x)-A_{i+1}(x)+\frac{2}{3}-A_{i}(x)+2 A_{i}^{2}(x) A_{i+1}(x)}{1-2 A_{i}(x) A_{i+1}(x)} \\
& =\frac{2 A_{i+1}^{2}(x)-1+6 A_{i}^{2}(x) A_{i+1}(x)}{3\left[1-2 A_{i}(x) A_{i+1}(x)\right]} \\
& =\frac{\left[1-2 A_{i}(x)\right]^{3}}{3\left[1-2 A_{i}(x) A_{i+1}(x)\right]}
\end{aligned}
$$

Then

$$
\begin{aligned}
A_{i+1}^{*}(x)-A_{i+1}(x) & =-\frac{\left[1-2 A_{i}(x)\right]^{3}}{3\left[1-2 A_{i}(x) A_{i+1}(x)\right]}, \\
\bar{S}_{T}(x)-F_{1}(x) & =\frac{\left[1-2 A_{i}(x)\right]^{3}}{3\left[1-2 A_{i}(x) A_{i+1}(x)\right]}\left(y_{i}-y_{i+1}\right) .
\end{aligned}
$$

Then

$$
\left|\bar{S}_{T}(x)-F_{1}(x)\right|=\frac{\left|\left[1-2 A_{i}(x)\right]^{3}\right|}{3\left[1-2 A_{i}(x) A_{i+1}(x)\right]}\left|y_{i+1}-y_{i}\right| \leq \frac{1}{3}\left|y_{i+1}-y_{i}\right| \leq \frac{1}{3}\left\|s^{\prime}\right\|_{\infty} h .
$$

(2)

$$
\begin{aligned}
C_{i+1}^{*}(x) & =\frac{A_{i}^{6}(x)+2 A_{i+1}^{2}(x)\left(A_{i}^{2}(x)+A_{i+1}^{2}(x)\right)^{2}}{3\left(A_{i}^{4}(x)+A_{i}^{2}(x) A_{i+1}^{2}(x)+A_{i+1}^{4}(x)\right)\left(A_{i}^{2}(x)+A_{i+1}^{2}(x)\right)} \\
A_{i+1}^{*}(x) & =\frac{A_{i+1}^{2}(x)}{A_{i}^{2}(x)+A_{i+1}^{2}(x)}
\end{aligned}
$$

then

$$
\begin{aligned}
C_{i+1}^{*}(x)-A_{i+1}^{*}(x)= & \frac{A_{i}^{6}(x)+2 A_{i+1}^{2}(x)\left(A_{i}^{2}(x)+A_{i+1}^{2}(x)\right)^{2}}{3\left(A_{i}^{4}(x)+A_{i}^{2}(x) A_{i+1}^{2}(x)+A_{i+1}^{4}(x)\right)\left(A_{i}^{2}(x)+A_{i+1}^{2}(x)\right)} \\
& -\frac{A_{i+1}^{2}(x)}{A_{i}^{2}(x)+A_{i+1}^{2}(x)} \\
= & \frac{\left(A_{i}^{2}(x)-A_{i+1}^{2}(x)\left(A_{i}^{4}(x)+A_{i+1}^{4}(x)\right)\right.}{\left.3\left(A_{i}^{4}(x)+A_{i}^{2}(x) A_{i+1}^{2}(x)\right)+A_{i+1}^{4}(x)\right)\left(A_{i}^{2}(x)+A_{i+1}^{2}(x)\right)} . \\
C_{i}^{*}(x)-A_{i}^{*}(x)= & -\left(C_{i+1}^{*}(x)-A_{i+1}^{*}(x)\right) .
\end{aligned}
$$


Then $\bar{S}_{L^{2}}(x)-F_{1}(x)=\left(C_{i+1}^{*}(x)-A_{i+1}^{*}(x)\right)\left(y_{i+1}-y_{i}\right)$. So

$$
\begin{aligned}
& \left|\bar{S}_{L^{2}}(x)-F_{1}(x)\right|=\left|C_{i+1}^{*}(x)-A_{i+1}^{*}(x)\right|\left|y_{i+1}-y_{i}\right| \\
& =\frac{\left|A_{i}^{2}(x)-A_{i+1}^{2}(x)\right|\left(A_{i}^{4}(x)+A_{i+1}^{4}(x)\right)}{\left.3\left(A_{i}^{4}(x)+A_{i}^{2}(x) A_{i+1}^{2}(x)\right)+A_{i+1}^{4}(x)\right)\left(A_{i}^{2}(x)+A_{i+1}^{2}(x)\right)}\left|y_{i+1}-y_{i}\right| \leq \frac{1}{3} \mid y_{i+1} \\
& \quad-y_{i} \mid \leq \frac{1}{3}\left\|s^{\prime}\right\|_{\infty} h .
\end{aligned}
$$

And

$$
\begin{aligned}
C_{i+1}^{*}(x)-A_{i+1}(x)= & C_{i+1}^{*}(x)-A_{i+1}^{*}(x)+A_{i+1}^{*}(x)-A_{i+1}(x) \\
= & \frac{\left(A_{i}^{2}(x)-A_{i+1}^{2}(x)\right)\left(A_{i}^{4}(x)+A_{i+1}^{4}(x)\right)}{3\left(A_{i}^{4}(x)+A_{i}^{2}(x) A_{i+1}^{2}(x)+A_{i+1}^{4}(x)\right)\left(A_{i}^{2}(x)+A_{i+1}^{2}(x)\right)} \\
& +\frac{A_{i}(x) A_{i+1}(x)\left[A_{i+1}(x)-A_{i}(x)\right]}{A_{i}^{2}(x)+A_{i+1}^{2}(x)} \\
= & \frac{\left[A_{i}^{2}(x)-A_{i+1}^{2}(x)\right]}{3\left[A_{i}^{2}(x)+A_{i+1}^{2}(x)\right]}\left[\frac{A_{i}^{4}(x)+A_{i+1}^{4}(x)}{A_{i}^{4}(x)+A_{i}^{2}(x) A_{i+1}^{2}(x)+A_{i+1}^{4}(x)}\right. \\
& \left.-3 A_{i}(x) A_{i+1}(x)\right] \\
= & \frac{\left[A_{i}^{2}(x)-A_{i+1}^{2}(x)\right]}{3\left[A_{i}^{2}(x)+A_{i+1}^{2}(x)\right]}\left[1-\frac{A_{i}^{2}(x) A_{i+1}^{2}(x)}{A_{i}^{4}(x)+A_{i}^{2}(x) A_{i+1}^{2}(x)+A_{i+1}^{4}(x)}\right. \\
& \left.-3 A_{i}(x) A_{i+1}(x)\right]
\end{aligned}
$$

Because of $\left|\frac{\left[A_{i}^{2}(x)-A_{i+1}^{2}(x)\right]}{3\left[A_{i}^{2}(x)+A_{i+1}^{2}(x)\right]}\right| \leq 1,1 \geq 1-\frac{A_{i}^{2}(x) A_{i+1}^{2}(x)}{A_{i}^{4}(x)+A_{i}^{2}(x) A_{i+1}^{2}(x)+A_{i+1}^{4}(x)}-$ $3 A_{i}(x) A_{i+1}(x) \geq 1-\frac{1}{3}-\frac{3}{4}=\frac{5}{12}>0$.

Thus $\left|C_{i+1}^{*}(x)-A_{i+1}(x)\right| \leq \frac{1}{3}$. So

$$
\left|\bar{S}_{L^{2}}(x)-F_{1}(x)\right|=\left|C_{i+1}^{*}(x)-A_{i+1}(x)\right|\left|y_{i+1}-y_{i}\right| \leq \frac{1}{3}\left\|s^{\prime}\right\|_{\infty} h
$$

Then $\left\|\bar{S}_{L^{2}}(x)-F_{1}(x)\right\|_{\infty} \leq \frac{1}{3}\left\|s^{\prime}\right\|_{\infty} h$.

Theorem 8 When $\bar{S}(x) \in\left\{\bar{S}_{T}(x), \bar{S}_{L^{2}}(x)\right\}$,

$$
\|s-\bar{S}\|_{\infty} \leq \frac{1}{8}\left\|s^{\prime \prime}\right\|_{\infty} h^{2}+\frac{1}{3}\left\|s^{\prime}\right\|_{\infty} h .
$$

\section{Proof}

$$
\|s-\bar{S}\|_{\infty} \leq\left\|s-F_{1}\right\|_{\infty}+\left\|\bar{S}-F_{1}\right\|_{\infty} \leq \frac{1}{8}\left\|s^{\prime \prime}\right\|_{\infty} h^{2}+\frac{1}{3}\left\|s^{\prime}\right\|_{\infty} h
$$



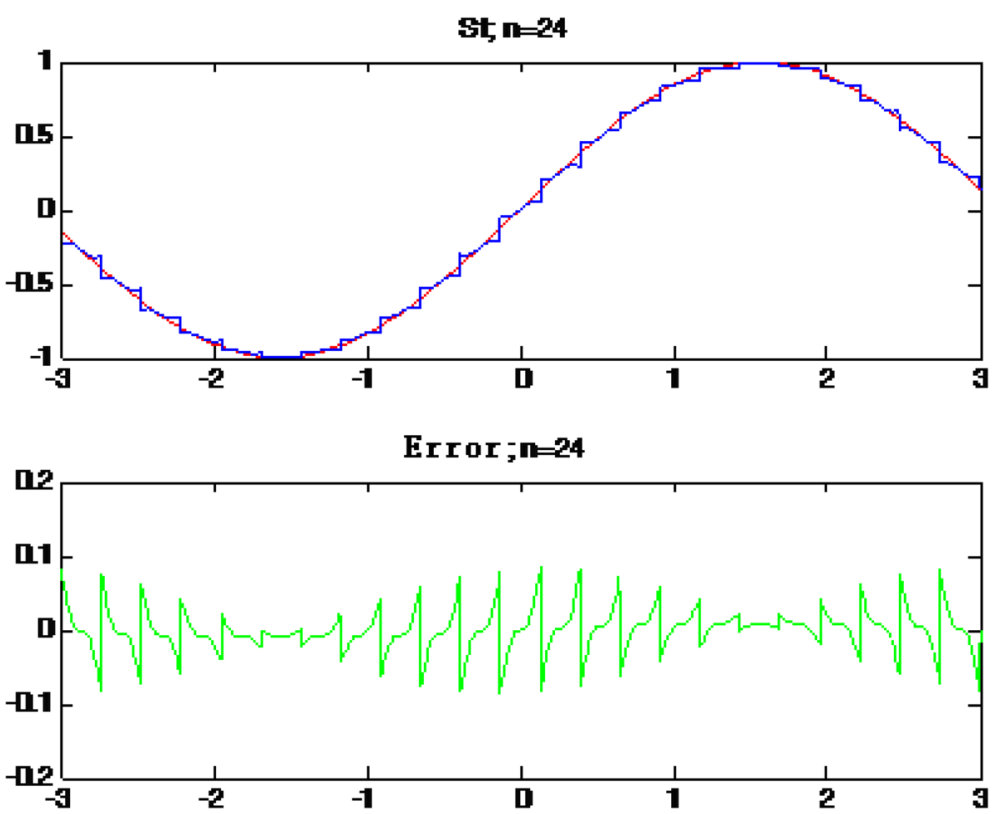

Fig. 1 The simulation result of $\bar{S}_{T}(x)$

Notes 3 (1) We get $\bar{S}_{T}(x)$ and $\bar{S}_{L^{2}}(x)$ are assumed $s(x)$ to be monotonic function. For non-monotonic function, we can divide $X$ into several intervals, and let $s(x)$ are monotonic in each interval. Because $\bar{S}_{T}(x)$ and $\bar{S}_{L^{2}}(x)$ can approximate $s(x)$ in each monotonic interval, $\bar{S}_{T}(x)$ and $\bar{S}_{L^{2}}(x)$ can approximate $s(x)$ in entire $X$. For example, let $s(x)=\sin x, X=[-\pi, \pi], X_{1}=\left[-\pi,-\frac{\pi}{2}\right], X_{2}=\left(-\frac{\pi}{2},-\frac{\pi}{2}\right), X_{3}=$ $\left[-\frac{\pi}{2},-\pi\right]$. Then $s(x)$ is monotonic function in $X_{1}, X_{2}, X_{3}$. If $\varepsilon=0.1$, then from Theorem 8 we can know that: $n=17$ in $X_{1}$ and $X_{3}, n=24$ in $X_{2}$. So we let $n=17 \times 2+33=67$, then $\bar{S}_{L^{2}}(x)$ can approximate $s(x)$ with error not more than 0.1 .

(2) From Theorem 8 we can know that: $\bar{S}_{T}(x)$ and $\bar{S}_{L^{2}}(x)$ have first-order approximation accuracy to $s$ and they have the same error estimates upper bound.

Example 1 Let $s(x)=\sin x, x \in[-3,3]$. Then $\left\|S^{\prime \prime}\right\|_{\infty}=\left\|S^{\prime}\right\|_{\infty}=1$.

If $\varepsilon=0.1$, from $\frac{1}{8}\left\|S^{\prime \prime}\right\| h^{2}+\frac{1}{3}\left\|S^{\prime}\right\|_{\infty} h<0.1$ we can know that $n=24$.

For $\varepsilon=0.1$, Figs. 1 and 2 show the simulation figure and the error estimates curves of $\bar{S}_{T}(x)$ and $\bar{S}_{L^{2}}(x)$ to $s$.

\section{Application Analysis}

The fuzzy trustworthiness theory and systems in this paper have wide application fields. It provides a completely new thought in theory and application study on such 

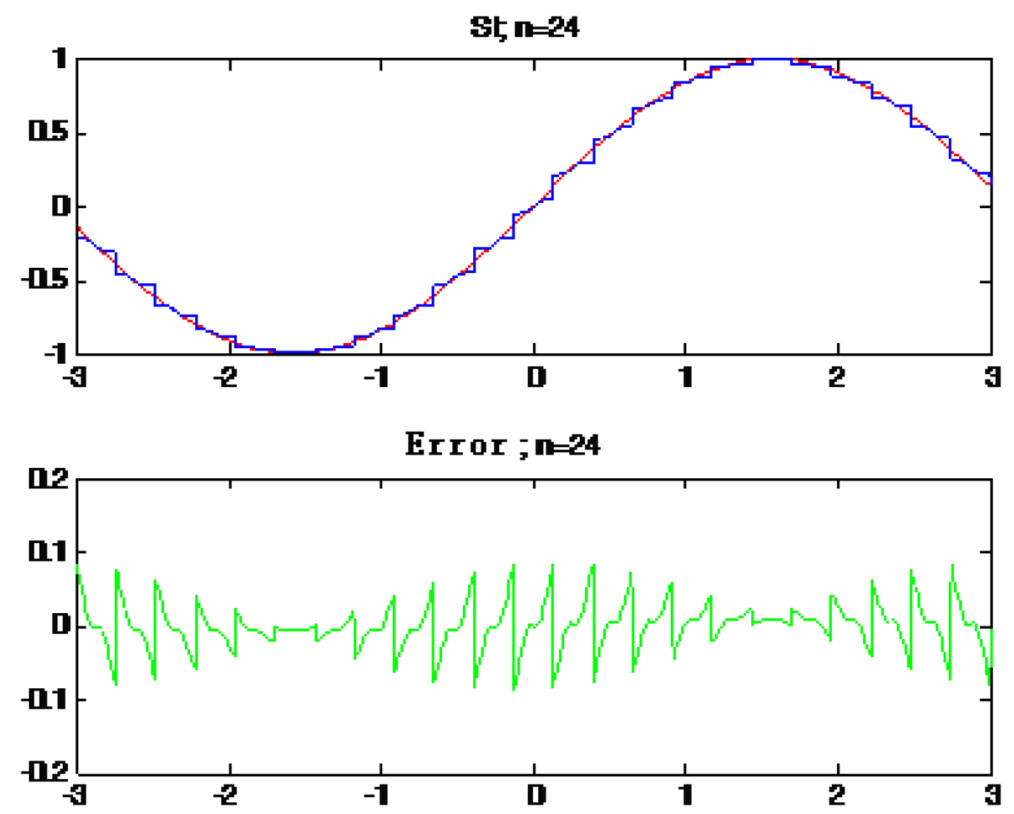

Fig. 2 The simulation result of $\bar{S}_{L^{2}}(x)$

fields as fuzzy logic and neural networks [20],factor neural networks [21-25], and fuzzy expert systems [26]. Fuzzy trustworthiness system, together with its theories, can be used in quantifiable description and measurement of trustworthy software [2731], and this would be a new direction of expand research on software trustworthiness metrics models. From the view of trustworthiness theory and fuzzy system, network attack and defence [31-34], engineering system automatic design process [35,36] and the design of fuzzy controller [37], etc., can also be explored, providing a new research method and direction for these fields.

\section{Conclusion}

This paper has researched bounded product implication and Larsen square implication and obtained two specific probability density functions. It is pointed out that these probability distributions have the same mathematical expectation and nearly the same variance and covariance although we have different probability density function expression by use of the different fuzzy implication operators. And we also got the center-of-gravity fuzzy trustworthiness systems of these two probability distributions, then we gave sufficient condition of the universal approximations for those fuzzy trustworthiness systems. As for application researches, fuzzy trustworthiness system can be applied in software trustworthiness metrics, network attack and defence, engineering system automatic design process and the design of fuzzy controller,etc. 
Acknowledgments This research was supported by National Natural Science Foundation of China (Major Research Plan, No.90818025).

\section{References}

1. Kai-yuan C, Chuan-yuan W, Ming-lian Z (1993) Basic concepts in fuzzy reliability theories. Acta Aeronaut et Astronaut Sin 14(7):388-398

2. WANG LX (1997) A course in fuzzy systems and fuzzy control. Prentice-Hall, Inc., Upper Saddle River

3. Zadeh LA (1975) The concept of a linguistic variable and its application to approximate reasoning I. Inf Sci 8(3):199-251

4. Zadeh LA (1975) The concept of a linguistic variable and its application to approximate reasoning II. Inf Sci 8(4):301-359

5. WANG GJ (1999) The triple I method with total inference rules of fuzzy reasoning. Sci China Ser E 29(1):43-53

6. Li H-X, You F, Peng J (2004) Fuzzy controllers based on some fuzzy implication operators and their response functions. Prog Nat Sci 14(1):15-20

7. Li H, Peng J, Wang J (2005) Fuzzy systems and their response functions based on commonly used fuzzy implication operators. Control Theory Appl 22(3):341-347

8. Li H, Peng J, Wang J (2005) Fuzzy systems based on triple I algorithm and their response ability. J Syst Sci Math Sci 25(5):578-590

9. Hou J, You F, Li HX (2005) Some fuzzy controllers based on the triple I algorithm and their response ability. Prog Nat Sci 15(1):29-37

10. Wang LX, Mendel JM (1992) Fuzzy basis functions, universal approximation and orthogonal least sequares learning. IEEE Trans Neural Netw 3(5):807-814

11. Zeng XJ, Singh MG (1994) Approximation theory of fuzzy systems-SISO case. IEEE Trans Fuzzy Syst 2(2):162-176

12. Zeng XJ, Singh MG (1995) Approximation theory of fuzzy systems-MIMO case. IEEE Trans Fuzzy Syst 3(2):219-235

13. Ying H (1994) Sufficient conditions on general fuzzy systems as function approximators. Automatica 30(3):521-525

14. Zeng K, Zhang N-Y, Xu W (2000) A comparative study on sufficient conditions for Takagi-Sugeno fuzzy systems as universal approximators. IEEE Trans Fuzzy Syst 8(6):773-780

15. Li YM, Shi ZK, Li ZH (2002) Approximation theory of fuzzy systems based upon genuine many-valued implication-SISO case. Fuzzy Sets Syst 130(2):147-157

16. Li YM, Shi ZK, Li ZH (2002) Approximation theory of fuzzy systems based upon genuine many-valued implication-MISO case. Fuzzy Sets Syst 130(2):159-174

17. Li D, Shi Z, Li Y (2008) Sufficient and necessary conditions for Boolean fuzzy systems as universial approximators. Inf Sci 178(2):414-424

18. Li H (1998) Interpolation mechanism of fuzzy control. Sci China Ser E 28(3):312-320

19. Li H (2006) Probability representation of fuzzy systems. Sci China Ser E 36(4):373-397

20. Liu Z, Liu Y (1996) Fuzzy logic and neural networks-a research to the basic theory. BUAA (Beihang University) Press

21. Liu Z, Liu Y (1992) The theory of factor neural networks and Its realizing strategy. Normal University Press, Beijing

22. Liu Z, Liu Y (1994) The theory of factor neural networks and its applications. Guizhou Science and Technology Publishing House

23. Liu Z (2010) Advances in factor neural network theory and its applications. In: 4th international conference of fuzzy information and engineering, Shomal University, Amol. 14-15 Oct 2010

24. Liu Z (1994) Method of factor neural networks to knowledge process. Fuzzy Syst Math

25. Liu Y, Liu Z (1994) A Method of factor neural networks. Fuzzy Syst Math

26. Liu Y, Liu Z (1995) Principle and design for fuzzy expert system, BUAA (Beihang University) Press

27. Tang Y, Liu Z (2010) Progress in software trustworthiness metrics models. Comput Eng Appl 46(27):12-16

28. Wang Y, Liu Z (2006) Risk assessment model for network security based on PRA. Comput Eng 32(1):40-42 
29. Liu X, Liu Z, Yu D (2007) Research on method of security management metrics based on hierarchy protection. Control Manag 45:39-40

30. Liu X, Liu Z, Yu D (2007) Method of security management metrics based on AHP model. Microcomput Inf 160:33-34

31. Wang X, Chen Y, Liu Z (2008) Component-based software developing method for group companies management information system; Source Wuhan University. J Nat Sci 13(1):37-44

32. Tao Y, Xia Y, Liu Z (2008) Research on intelligent simulation model of network defense[A]. In: Computational intelligence and industrial application PACIIA

33. Tao Y, Liu Z, Zhang Z, Wang P, Guo C (2010) Research on network attack situation niching model based on FNN theory. Chin High Technol Lett 20(7):680-684

34. Xu P, Hou Z, Liu Z (2011) Driving and control of torque for direct-wheel-driven electric vehicle with motors in serial. Expert Syst Appl 38(1):80-86

35. Liu Z, Liu Y (1987) Application of the fuzzy set theory in the engineering system automatic design process. Int Fuzzy Syst Knowl Eng 2:388-396

36. Liu Z, Liu Y (1987) Some possible Application of the fuzzy set theory in the engineering system automatic design process. In: Proceedings of SICE' 87 in Hiroshima

37. Liu Z (1989) The fuzzy inference of programmable fuzzy controller. In: Paper of 19th international symposium on ML 